\title{
Synthesis of isoindolinones by Pd-catalyzed coupling between $N$-methoxybenzamide and styrene derivatives
}

Changkun Xia, ${ }^{\dagger}$ Andrew J. P. White and King Kuok (Mimi) Hii*

Department of Chemistry, Imperial College London, Exhibition Road, South Kensington, London SW7 2AZ, U.K.*E-mail: mimi.hii@imperial.ac.uk.

${ }^{\dagger}$ Current affiliation: School of Chemistry and Chemical Engineering, Jiangsu University, Zhenjiang, 212013, China.

Table of Contents graphic

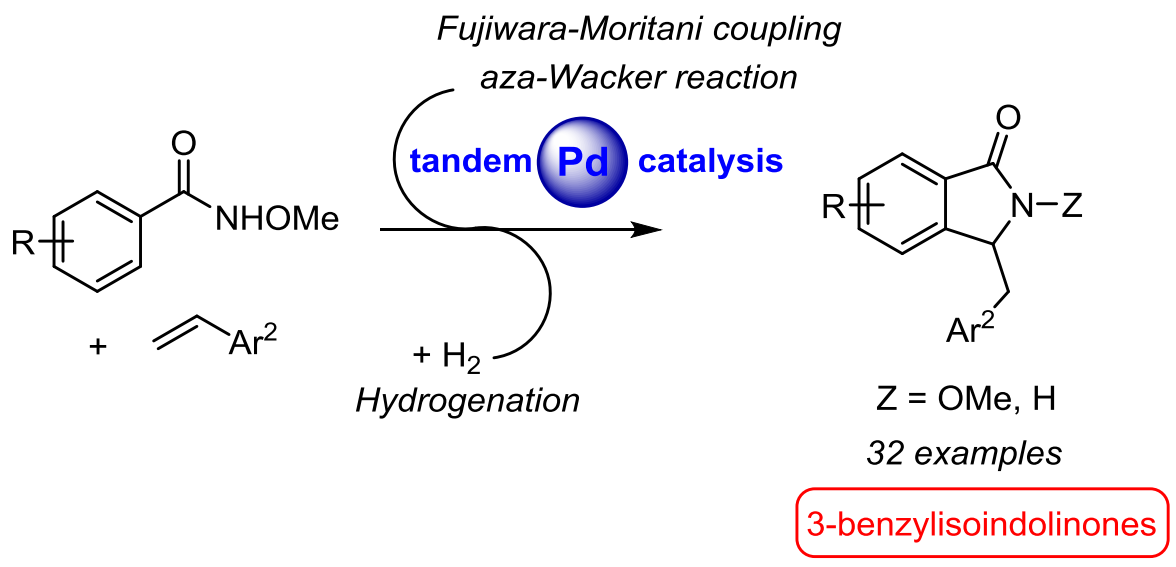

\begin{abstract}
An atom-economical protocol for a tandem process involving Fujiwara-Moritani-aza-Wacker reactions has been developed for the Pd-catalyzed coupling between $N$-methoxy benzamide with styrene derivatives. The generality of the methodology was demonstrated by the synthesis of a library of twentyfive 3-benzylidene isoindolinones in moderate to good yields. A further twenty-two 3-benzyl derivatives were obtained by telescoping the process with a catalytic hydrogenation reaction.
\end{abstract}




\section{Introduction}

3-Benzyl-substituted-isodolinones (I) constitute a family of privileged pharmacophores (Fig. 1) with significant therapeutic potentials, such as glycine transporter (GlyT1) inhibitors (Abbvie), ${ }^{1}$ and aldosterone synthase (CYP11B2 or CYP11B1) inhibitors (Hoffmann-la Roche). ${ }^{2}$ The sub-structure is also found in many natural products, including isoindolobenzazepine alkaloids (e.g. lennoxamine ${ }^{3}$ ), and aristolactam alkaloids (including piperolactams ${ }^{4}$ ), known to have potent anti-tumor activities. ${ }^{5}$

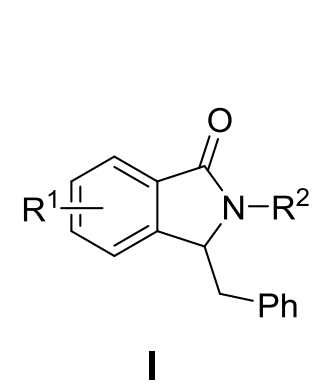

GlyT1 inhibitors CYP11B1/2 inhibitors

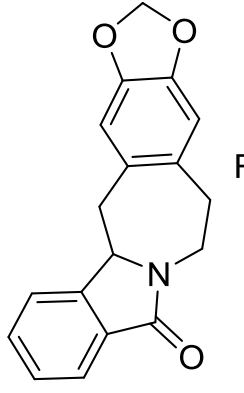

lennoxamine<smiles>[R10]Oc1ccc2c(c1O[R10])-c1c(cc([R2])c([R20])c1O[R1])C(=O)NC2C</smiles>

Aristolactam alkaloids

Figure 1. Biologically active 3-benzyl-substituted isoindolinones (I) and natural products containing the sub-structure.

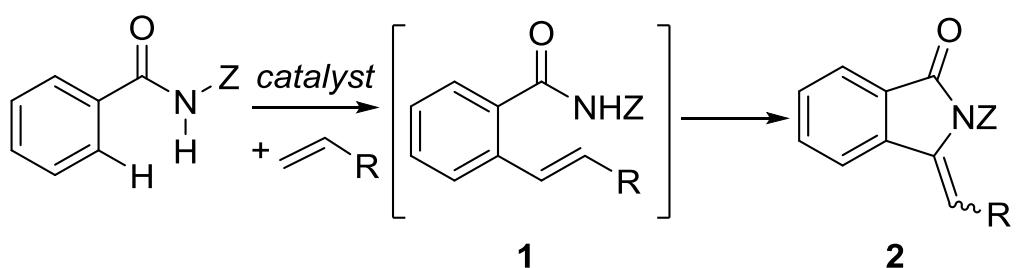

Scheme 1. Proposed route for assembling $\mathbf{I}$.

Conceptually, structure I can be constructed in two steps: By a Fujiwara-Moritani (FM) reaction between benzamide and styrene derivatives, followed by an intramolecular $a z a$-Wacker reaction (Scheme 1); the resultant product $\mathbf{2}$ can then be subjected to catalytic hydrogenation to yield the target structure. The FM reaction between a benzamide derivative and an alkene can be achieved using $\mathrm{Rh},{ }^{6} \mathrm{Ru}^{7}$ and $\mathrm{Ir}^{8}$ catalysts . 
However, using these catalysts, the reaction terminates at the formation of the acyclic compound (1). In contrast, both reactions can be effected in tandem under Pd catalysis to give the $N$-heterocycle 2 as the final product. This was first reported independently by the research groups of Wrigglesworth et $a l^{9}$ and Li et al in $2011,{ }^{10}$ when $\mathrm{Pd}(\mathrm{OAc})_{2}$ were used to catalyze the coupling between $N$-methoxybenzamides with activated alkenes containing electron-withdrawing substituents, i.e. $\mathrm{R}=\mathrm{CO}_{2} \mathrm{R}, \mathrm{CONR}_{2}, \mathrm{SO}_{2} \mathrm{Ph}_{\text {, }}$ COR. One example using styrene as the alkene reactant was reported to afford the 2-benzylidene derivative $(\mathrm{R}=\mathrm{Ph}, \mathbf{2 a a})$ in a moderate yield $(\leq 50 \%)$; notably, no further examples of other conjugated alkenes were provided. In this work, we have modified the catalytic protocol to widen the scope of this methodology to a wide range of styrene derivatives $(Z=A r)$, with the aim of synthesizing a library of 3benzyl substituted isoindolinones (analogues of compound I) for biological evaluation (Scheme 2).

Previous work [ref. 9, 10]:

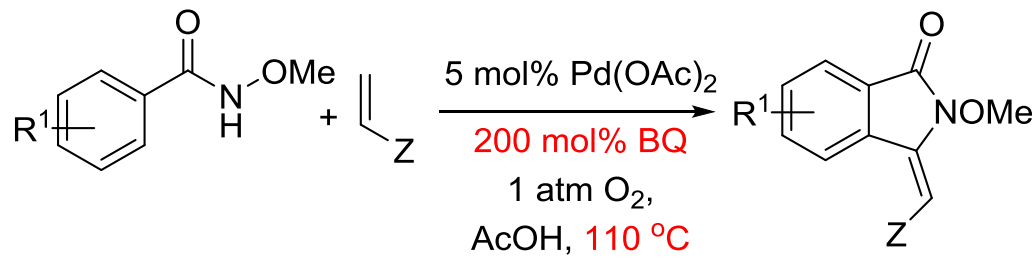

$Z=$ EWG (>30 examples)

$\mathrm{Z}=\mathrm{Ph}$, only one example, $50 \%$

This work:

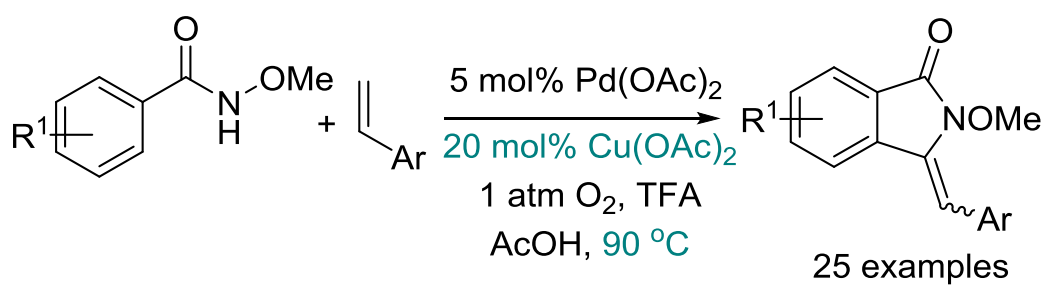<smiles>[Z]N1C(=O)C2=C(C=C[R1]C=C2)C1C[Te]</smiles>

22 examples

Scheme 2. Modification of the tandem FM- $a z a$-Wacker reactions for the synthesis of $\mathbf{I}$. 


\section{Results and discussion}

$N$-Methoxybenzamide and styrene were initially employed as model substrates in the evaluation of reaction parameters (Table 1). Following the reported procedure by Li et al, ${ }^{10}$ palladium(II) acetate was employed as a catalyst precursor with 2 equivalents of the oxidant (benzoquinone, BQ), and the reaction was performed in acetic acid at $100^{\circ} \mathrm{C}$. This afforded the expected product $\mathbf{2}$ aa in $27 \%$ yield (entry 1 ). This was lower than the reported yields of $46 \%$, which may be due to the prolonged reaction time (48 $\mathrm{h}$ vs $10 \mathrm{~h}$ ). Subsequent studies at lower reaction temperatures did not lead to any improvement (entry 2), and the addition of TFA only has a marginally beneficial effect under these conditions (entry 3). By increasing the amount of oxidant and styrene, the product yield may be improved (entry 4), which also allowed the reaction time to be shortened (entry 5).

Notably, attempt to lower the amount of oxidant from 2 equivalents to 1 equivalent adversely affected the reaction, even in the presence of $\mathrm{O}_{2}$ (entry 6), i.e. we were unable to replicate the $50 \%$ yield achieved by using $20 \mathrm{~mol} \%$ of BQ, as reported by Wrigglesworth et al. ${ }^{9}$ The need to use an excess of BQ is a significant issue; apart from poor atom-economy, benzoquinone is also genotoxic, ${ }^{11}$ which may interfere with subsequent biological testing should any residue remain in the product. Therefore, although a reasonable yield (69\%) can be achieved using this oxidant, it was decided that a safer alternative should be sought. With this in mind, a number of more benign oxidants were evaluated, including potassium and ammonium persulfates, as well as tert-butyl perbenzoate (Table S1, Supporting information). The use of these strong oxidants was found to be incompatible with the benzamide substrates, causing them to decompose at temperatures $>50^{\circ} \mathrm{C}$. After some further investigation, we were able to identify $\mathrm{O}_{2} / 20 \mathrm{~mol} \%$ $\mathrm{Cu}(\mathrm{OAc})_{2}$ as a good replacement of benzoquinone, delivering $\mathbf{2}$ aa at a slightly elevated temperature of 90 ${ }^{\circ} \mathrm{C}$ (entries 7-8); in this case, the beneficial effect of TFA was more pronounced (entries 8 vs 9), as it suppressed the competitive hydrolysis of $N$-methoxybenzamide under these conditions. 
Table 1. Initial evaluation of reaction parameters. ${ }^{a}$
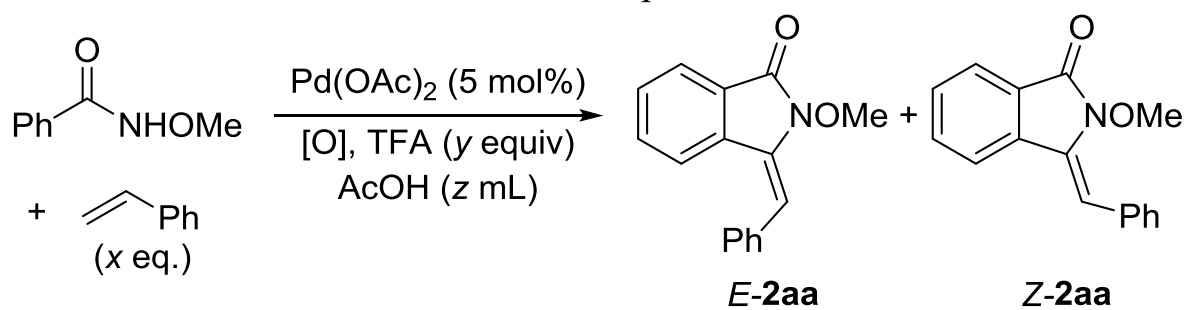

\begin{tabular}{|c|c|c|c|c|c|c|c|}
\hline Entry & {$[\mathrm{O}]^{b}$} & $x$ & $y$ & $z$ & $\mathrm{~T}\left({ }^{\circ} \mathrm{C}\right)$ & $\mathrm{t}(\mathrm{h})$ & Yield $^{c}(\%)$ \\
\hline 1 & BQ (2) & 2 & - & 3 & 100 & 48 & 27 \\
\hline 2 & BQ (2) & 2 & - & 3 & 80 & 48 & 30 \\
\hline 3 & BQ (2) & 2 & 1 & 3 & 70 & 48 & 34 \\
\hline 4 & BQ (2.5) & 3 & 1 & 3 & 70 & 48 & 69 \\
\hline 5 & BQ (2.5) & 3 & 1 & 1.5 & 70 & 24 & 66 \\
\hline 6 & $\mathrm{BQ}(1), \mathrm{O}_{2}$ & 2 & 1 & 3 & 70 & 48 & 34 \\
\hline 7 & $\mathrm{Cu}(\mathrm{OAc})_{2}(0.2), \mathrm{O}_{2}$ & 3 & 1 & 1.5 & 80 & 24 & 48 \\
\hline 8 & $\mathrm{Cu}(\mathrm{OAc})_{2}(0.2), \mathrm{O}_{2}$ & 3 & 1 & 1.5 & 90 & 24 & 60 \\
\hline 9 & $\mathrm{Cu}(\mathrm{OAc})_{2}(0.2), \mathrm{O}_{2}$ & 3 & - & 1.5 & 90 & 24 & 44 \\
\hline 10 & $\mathrm{Cu}(\mathrm{OAc})_{2}(0.2), \mathrm{O}_{2}$ & 3 & 1 & 1.5 & 100 & 24 & 50 \\
\hline
\end{tabular}

${ }^{\bar{a} \text { General conditions: Reactions were performed using } N \text {-methoxy-benzamide as the limiting reagent }(0.3}$ $\mathrm{mmol}$ ). More results are listed in Tale S1 (Supporting information). ${ }^{b}$ Equiv indicated in parenthesis. 'Isolated yield after column chromatography.

In earlier work, ${ }^{9,10} \mathbf{2 a a}$ was reported to be formed exclusively as an $E$-isomer. In the present study, however, it was obtained as a mixture of isomers, irrespective of the oxidant or reaction conditions, 
typically in a ratio of approximately $2: 1$. This was established by integrating the methoxy proton resonance at 4.1 and $3.5 \mathrm{ppm}$, respectively. The configuration isomers were subsequently separated by column chromatography, and the $E$-configuration of the major isomer was confirmed by X-ray crystallography (Figure S1, Supporting Information). In the solid state structure, the pendant phenyl is twisted out-of-plane from the isoindolinone ring, in order to avoid unfavourable interactions between the ortho H's.

Under these reaction conditions, polymerization of styrene is a minor competitive process. However, the NMR spectrum of the reaction mixture contained additional resonance peaks, indicating the presence of a persistent side product ( $c a .20 \%$ ), identified by a distinct ${ }^{1} \mathrm{H}$ NMR singlet signal at $8.51 \mathrm{ppm}$. A small amount of this impurity was isolated. Its ${ }^{1} \mathrm{H}$ NMR spectrum, with the accompanying mass ion $\left(\mathrm{MH}^{+}\right)$of 176, are consistent with the formation of the 6-membered $N$-methoxy isoquinolinone 3 (Scheme 3 ). ${ }^{12}$ The formation of this unexpected side product is attributed to the formation of the minor $Z$-isomer of 1aa, which undergoes 6-endo-trig cyclization in the subsequent $a z a$-Wacker reaction (anti-addition). In the absence of an accessible $\beta$-hydride, the putative intermediate (A) undergoes a syn-coplanar $\beta$-phenyl elimination to afford compound 3. Such $\beta$-aryl elimination processes involving $\mathrm{C}$-C cleavage are rare; known only to occur in reactions involving sterically bulky arylmethanols. ${ }^{13}$ 


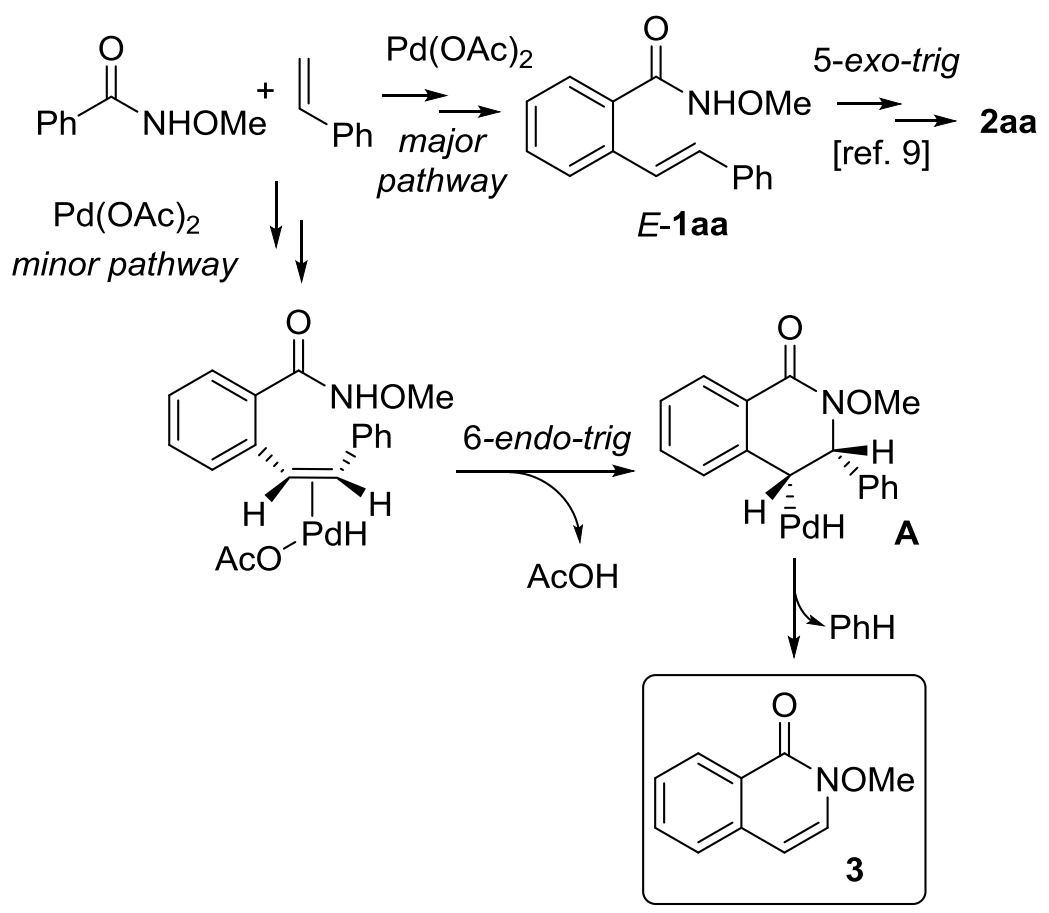

Scheme 3. Competitive formation of side product 3 .

Next, the generality of the catalytic methodology was tested. Using the new protocol, a small library of twenty-five new 3-benzylidene isoindolinone derivatives was constructed from readily-available benzamide and styrene derivatives (Table 2). Reaction yields are moderate to good, ranging between 49$72 \%$. In general, better yields were obtained with styrene derivatives bearing electron-withdrawing substituents. Again, in all cases, a mixture of $E / Z$-isomers was obtained, typically in a 2:1 ratio in favor of the $E$-isomer. The $E$-configuration is favored when 2-methyl- or 2-methoxy-substituted $N$-methoxybenzamides (entries 13-21) were used as substrates; particularly in combination with 2-vinyl naphthalene (entries 5, 11, 16 and 21). 
Table 2. Tandem reaction between $N$-methoxybenzamide and styrene derivatives. ${ }^{a}$

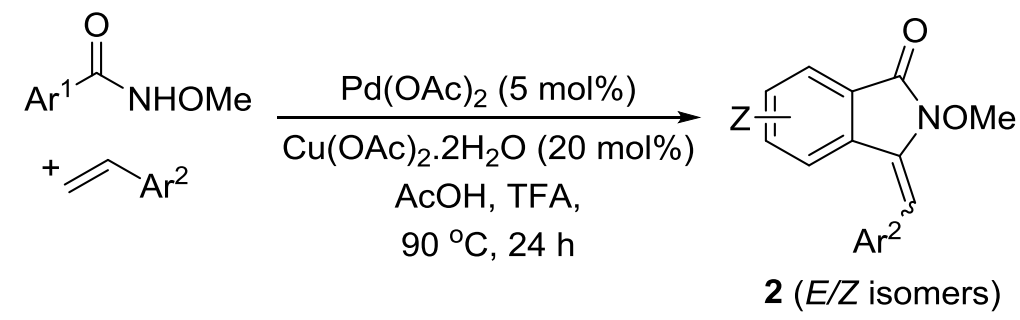

\begin{tabular}{|c|c|c|c|c|}
\hline Entry & $\mathrm{Ar}^{1}, \mathrm{Ar}^{2}$ & Product & Yield $(\%)^{b}$ & $E / Z$ \\
\hline 1 & $\mathrm{Ph}, \mathrm{Ph}$ & $\mathbf{2} \mathbf{a a}^{c}$ & 60 & $2 / 1$ \\
\hline 2 & $\mathrm{Ph}, 4-\mathrm{Cl}-\mathrm{Ph}$ & $\mathbf{2} \mathbf{a} \mathbf{b}^{c}$ & 68 & $2.1 / 1$ \\
\hline 3 & $\mathrm{Ph}, 4-\mathrm{F}-\mathrm{Ph}$ & $2 \mathrm{ac}$ & 53 & $2 / 1$ \\
\hline 4 & $\mathrm{Ph}, 4-\mathrm{Me}-\mathrm{Ph}$ & 2ad & 53 & $5 / 1$ \\
\hline 5 & $\mathrm{Ph}, 2-\mathrm{Nap}$ & $2 \mathbf{a e}$ & 59 & $2.3 / 1$ \\
\hline 6 & $\mathrm{Ph}, 4-\mathrm{CF}_{3}-\mathrm{Ph}$ & 2af & 72 & $2.4 / 1$ \\
\hline 7 & 4-Me-Ph, $\mathrm{Ph}$ & $2 d a$ & 63 & $1.9 / 1$ \\
\hline 8 & 4-Me-Ph, 4-Cl-Ph & $\mathbf{2} \mathbf{d} \mathbf{b}^{c}$ & 61 & $1.9 / 1$ \\
\hline 9 & 4-Me-Ph, 4-F-Ph & $2 d c$ & 71 & $1.5 / 1$ \\
\hline 10 & 4-Me-Ph, 4-Me-Ph & 2dd & 65 & $1.9 / 1$ \\
\hline 11 & 4-Me-Ph, 2-Nap & $2 \mathrm{de}$ & 66 & $3.5 / 1$ \\
\hline 12 & 4-Me-Ph, 4- $\mathrm{CF}_{3}-\mathrm{Ph}$ & $2 d f$ & 70 & $1.8 / 1$ \\
\hline 13 & 2-Me-Ph, $\mathrm{Ph}$ & 2ga & 69 & $2.7 / 1$ \\
\hline 14 & 2-Me-Ph, 4-Cl-Ph & $2 \mathbf{g b}^{c}$ & 62 & $3.1 / 1$ \\
\hline 15 & 2-Me-Ph, 4-Me-Ph & 2gd & 58 & $2.9 / 1$ \\
\hline 16 & 2-Me-Ph, 2-Nap & $2 g e$ & 68 & $3.5 / 1$ \\
\hline 17 & 2-Me-Ph, 4- $\mathrm{CF}_{3}-\mathrm{Ph}$ & $2 \mathrm{gf}$ & 59 & $2.5 / 1$ \\
\hline 18 & 2-MeO-Ph, $\mathrm{Ph}$ & 2ha & 58 & $4.3 / 1$ \\
\hline 19 & 2-MeO-Ph, 4-Cl-Ph & $\mathbf{2 h} \mathbf{b}^{c}$ & 63 & $3.1 / 1$ \\
\hline 20 & 2-MeO-Ph, 4-Me-Ph & 2hd & 52 & $4.2 / 1$ \\
\hline 21 & 2-MeO-Ph, 2-Nap & 2he & 48 & $3.7 / 1$ \\
\hline 22 & 4-MeO-Ph, 4-Cl-Ph & $2 \mathbf{i b}^{c}$ & 49 & $1.9 / 1$ \\
\hline 23 & 4-MeO-Ph, 4-Me-Ph & $2 \mathrm{id}$ & 55 & $1.7 / 1$ \\
\hline 24 & 4-MeO-Ph, 4- $\mathrm{CF}_{3}-\mathrm{Ph}$ & 2 if & 46 & $1.8 / 1$ \\
\hline 25 & 4- $\mathrm{CF}_{3}-\mathrm{Ph}, 4-\mathrm{Cl}-\mathrm{Ph}$ & $2 \mathbf{j} \mathbf{b}^{c}$ & 17 & $1 / 0$ \\
\hline
\end{tabular}


${ }^{a}$ Reaction conditions: $N$-methoxybenzamide ( $0.3 \mathrm{mmol}, 1$ equiv), styrene ( $0.9 \mathrm{mmol}, 3$ equiv), $\mathrm{Pd}(\mathrm{OAc})_{2}$ (0.015 mmol, $5 \mathrm{~mol} \%), \mathrm{Cu}(\mathrm{OAc}){ }_{2} \cdot{ }_{2} \mathrm{H}_{2} \mathrm{O}(0.06 \mathrm{mmol}, 20 \mathrm{~mol} \%)$, acetic acid $(1.5 \mathrm{~mL})$, trifluoroacetic acid (TFA, $0.3 \mathrm{mmol}, 1$ equivalent), $\mathrm{O}_{2}$ balloon, $90{ }^{\circ} \mathrm{C}$. ${ }^{b}$ Isolated yield obtained after column chromatography, $E: Z$ ratio indicated in parenthesis. ${ }^{c}$ Characterized by NMR spectroscopy after column chromatography.

In some cases, the $E / Z$ isomers may be separated by column chromatography for characterization

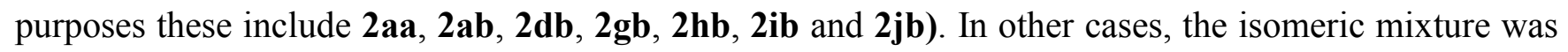
directly subjected to hydrogenation reactions over $\mathrm{Pd} / \mathrm{C}$ in a telescoped process, affording twenty-two novel 2-benzyl-substituted isoindolinones (Fig. 2). The hydrogenation reaction is known to proceed well in high yields for similar benzylideneisoindolidinones, when the exocyclic alkene contains an electronwithdrawing ester group. ${ }^{9,14}$ In the present case, the reduction of aryl-substituted 2required slightly elevated temperatures of between $40-50^{\circ} \mathrm{C}$. This was attributed to the twisted alkene moiety observed in the X-ray crystal structure (See Supporting Information), sterically hindering the accessibility to surfaceadsorbed hydrides. Under these conditions, the substrate may be reduced further to the $N$-demethoxylated product 4'. Interestingly, the outcome of the catalytic hydrogenation process is rather dependent upon the nature of the pendant aryl group. Notably, 4' was obtained as the major product when the aryl group contains fluorinated substituents. Conversely, the yields of chloro-substituted compounds (4ab, $\mathbf{4 g b}, \mathbf{4 d b}$ and 4ib) were also affected by the competitive dehalogenation of the chloride during the catalytic hydrogenation process. In this part of the work, X-ray crystallographic structures of reduced 4aa and 4aa' were obtained (Figures S2 and S3, Supporting Information), providing additional information of their structure features that may be useful for fragment-based discovery. 


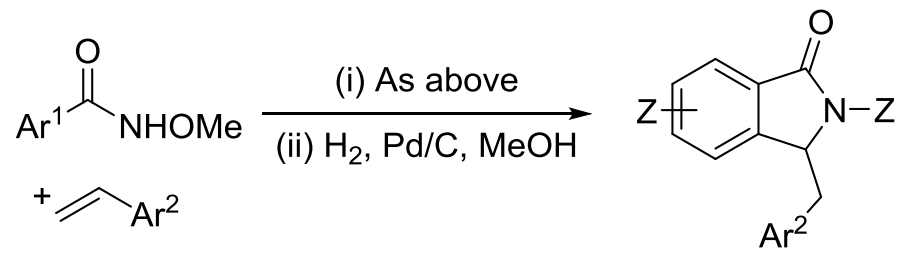

$$
Z \text { = OMe (4), H (4') }
$$

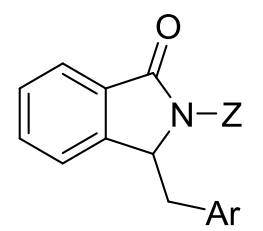

$\mathrm{Ar}=\mathrm{Ph}, \mathrm{aa}, \quad 47 \%(1.9: 1)$

$\mathrm{Ar}=4-\mathrm{Cl}-\mathrm{Ph}, \mathbf{a b}, 25 \%(1: 0)$

$\mathrm{Ar}=4-\mathrm{F}-\mathrm{Ph}, \mathrm{ac}, \quad 37 \%(1: 2)$

$\mathrm{Ar}=4-\mathrm{Me}-\mathrm{Ph}$, ad, $38 \%$ (5.3:1)

$\mathrm{Ar}=2-\mathrm{Nap}, \mathrm{ae}, \quad 43 \%(2: 1)$

$\mathrm{Ar}=4-\mathrm{CF}_{3}-\mathrm{Ph}$, af, $61 \%(1: 6.6)$

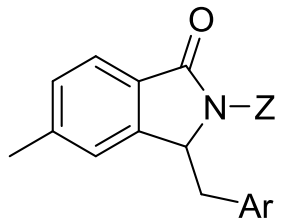

$\mathrm{Ar}=\mathrm{Ph}, \mathrm{da}, \quad 53 \%(1.4: 1)$

$\mathrm{Ar}=4-\mathrm{Cl}-\mathrm{Ph}, \mathbf{d b}, \quad 22 \%(1: 0)$

$\mathrm{Ar}=4-\mathrm{F}-\mathrm{Ph}, \mathrm{dc}, \quad 37 \%(1.8: 1)$

$\mathrm{Ar}=4-\mathrm{Me}-\mathrm{Ph}, \mathbf{d d}, \quad 36 \%(2.6: 1)$

$\mathrm{Ar}=2-\mathrm{Nap}$, de, $\quad 45 \%(1.8: 1)$<smiles>[Z]N1C(=O)c2cc(C)ccc2C1C[Al]</smiles>

$\mathrm{Ar}=\mathrm{Ph}, \mathbf{g a}, 57 \%(1.5: 1)$

$\mathrm{Ar}=4-\mathrm{Cl}-\mathrm{Ph}, \mathbf{g b}, 26 \%(1: 0)$

$\mathrm{Ar}=4-\mathrm{Me}-\mathrm{Ph}, \mathbf{g d}, \quad 56 \%(5.2: 1)$

$\mathrm{Ar}=2-\mathrm{Nap}, \mathbf{g e}, \quad 43 \%$ (5.1:1)

$\mathrm{Ar}=4-\mathrm{CF}_{3}-\mathrm{Ph}, \mathbf{g f}, 53 \%(1: 1.3)$

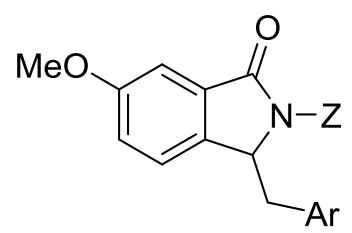

$\mathrm{Ar}=\mathrm{Ph}, \mathbf{h a}, \quad 52 \%(1: 1.3)$

$\mathrm{Ar}=4-\mathrm{Me}-\mathrm{Ph}, \mathrm{hd}, \quad 47 \%(3.7: 1)$

$\mathrm{Ar}=2-\mathrm{Nap}$, he, $\quad 44 \%$ (3.9:1)

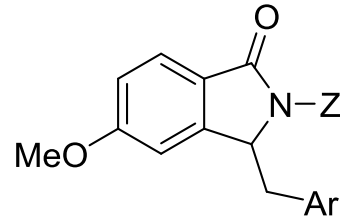

$\mathrm{Ar}=4-\mathrm{Cl}-\mathrm{Ph}, \mathbf{i b}, \quad 19 \%(1: 0)$

$\mathrm{Ar}=4-\mathrm{Me}-\mathrm{Ph}$, id, $40 \%(2.3: 1)$

$\mathrm{Ar}=4-\mathrm{CF}_{3}-\mathrm{Ph}$, if, $51 \%(5.4: 1)$

Figure 2. Reduced isoquinolines synthesized via telescoped steps (reported yields were calculated over two steps). Ratio of 4:4' is indicated in parenthesis.

\section{Conclusion}

The scope of the Pd-catalyzed tandem Fujiwara-Moritani (FM)-intramolecular $a z a$-Wacker cyclisation has been substantially expanded to styrene derivatives, replacing the use of excess genotoxic benzoquinone by benign $\mathrm{O}_{2}$, in the presence of $\mathrm{Cu}(\mathrm{OAc})_{2}$ as co-catalyst. By telescoping the process with catalytic hydrogenation, a series of 3-benzyl-substituted isoindolinones can be prepared from readily 
available $N$-methoxybenzamide and styrene derivatives in two synthetic operations. The methodology has good generality, providing access to $>40$ novel isoindolinone structures containing either a benzylidene or a benzyl substituent at position-3.

\section{Experimental Section}

\section{General Experimental Methods.}

Unless otherwise stated, all precursors were obtained from commercial suppliers and used as received without purification. Solvents were dried by passing through the columns of molecular sieves in a solvent purification system. All reactions were carried out in oven-dried glassware. Reaction temperatures are reported as the temperature of the bath/heating block surrounding the vessel unless otherwise stated. Analytical thin layer chromatography was performed on silica plates. Visualization was accomplished with UV light $(254 \mathrm{~nm})$, and/or $\mathrm{KMnO}_{4}$ staining solutions followed by heating. Column chromatography was performed on flash silica gel (40-63 mesh). ${ }^{1} \mathrm{H}$ and ${ }^{13} \mathrm{C}$ NMR spectra were recorded at ambient temperature $\left(25^{\circ} \mathrm{C}\right)$ on spectrometers. Residual protic solvents were used as an internal standard and ${ }^{13} \mathrm{C}$ resonances were referenced to the deuterated carbon. Chemical shifts $(\delta)$ are reported in ppm. Monoisotopic values for chlorinated compounds were reported and calculated using the major isotope $\left({ }^{35} \mathrm{Cl}\right)$.

Single crystals of E-2aa, 4aa and 4'aa suitable for X-ray crystallography was obtained as needles by recrystallization from EtOAc and hexane at room temperature. Selected crystals were centered on an Xray crystal structural data were collected using a diffractometer equipped with a CCD detector and a graphite monochromated Mo K $\alpha$ radiation $(\lambda=0.71073 \AA)$. The data collection routine, unit cell refinement, and data processing were carried out with the program CrysAlis. ${ }^{15}$ Structures were solved by the direct method and refined by full-matrix least-squares methods with SHELXL-97 programs. ${ }^{16} \mathrm{X}$-ray crystal data and structure refinement parameters were listed in Table S2 (Supporting information). The 
data were deposited at the CCDC database, with the reference numbers $1472315-1472317$ for $E$-2aa, 4aa, and 4'aa, respectively.

$N$-Methoxy benzamide derivatives (precursors for the catalytic reaction) were prepared from commercially available benzoyl chloride and $O$-methylhydroxylamine, by a published procedure. ${ }^{17}$ The characterization data of these compounds are entirely consistent with the literature values. ${ }^{17}$

General procedure for the tandem Fujiwara-Moritani-aza-Wacker reaction. A reduced volume Radley's reaction tube was charged with a stir bar, the appropriate styrene ( 0.9 mmol, 3 equiv), and $N$ methoxybenzamide $\left(0.3 \mathrm{mmol}, 1\right.$ equiv) derivatives, $\mathrm{Pd}(\mathrm{OAc})_{2}(0.05$ equiv $)$ and $\mathrm{Cu}(\mathrm{OAc})_{2} \cdot 2 \mathrm{H}_{2} \mathrm{O}(0.2$ equiv) and acetic acid $(1.5 \mathrm{~mL})$ at room temperature. The tube was evacuated briskly under vacuum and recharged with oxygen three times. The $\mathrm{O}_{2}$ atmosphere in the tube was maintained by connecting a balloon of $\mathrm{O}_{2}$ to the reaction tube through a needle pierced through the septum. The reaction vessel was then placed in an aluminum heating block heated to $90{ }^{\circ} \mathrm{C}$ and stirred. The reaction was monitored by TLC until the starting material was consumed. The reaction mixture was concentrated under vacuum and the residue was subjected directly to column chromatography using petroleum ether/EtOAc (6/1) as the eluent. A selection of the $Z$ - and $E$-isomer may be collected as separate fractions for characterization purposes, or as a combined fraction for the telescoped process.

The reaction can be successfully replicated on larger scale using $N$-methoxybenzamide (136 mg, 0.9 mmol, 1 equiv), styrene $\left(281 \mathrm{mg}, 2.7 \mathrm{mmol}, 3\right.$ equiv), $\mathrm{Pd}(\mathrm{OAc})_{2}(0.05$ equiv $)$ and $\mathrm{Cu}(\mathrm{OAc})_{2} \cdot 2 \mathrm{H}_{2} \mathrm{O}(0.2$ equiv), TFA (1 equiv), and acetic acid (4.5 mL). Following column chromatography, $43 \mathrm{mg}(19 \%)$ of the $Z$ - isomer and $91 \mathrm{mg}(40 \%)$ of the $E$-isomer were isolated separately.

Catalytic hydrogenation of 2 to 4 . A mixture of $Z / E-2$ was dissolved in anhydrous methanol (10 mL) in a Radley's reaction tube. $10 \mathrm{wt} \% \mathrm{Pd} / \mathrm{C}$ was added to this mixture before the system was then sealed with a cap and a balloon of hydrogen attached via a needle pierced through the septum. The reaction vessel 
was placed in the aluminum block and the mixture was stirred and heated between $40-50{ }^{\circ} \mathrm{C}$ for $2 \mathrm{~h}$. The reaction mixture was filtered through Celite and the filtrate concentrated to give a yellow solid which was then purified by flash column chromatography (petroleum ether /EtOAc, 4/1).

(Z)-3-Benzylidene-2-methoxyisoindolin-1-one, Z-2aa. Light yellow solid (15 mg, $0.059 \mathrm{mmol,} 20 \%$ ). ${ }^{1} \mathrm{H} \mathrm{NMR}\left(\mathrm{CDCl}_{3}, 400 \mathrm{MHz}\right): \delta 7.87-7.84(\mathrm{~m}, 1 \mathrm{H}), 7.75-7.72(\mathrm{~m}, 1 \mathrm{H}), 7.62(\mathrm{td}, 1 \mathrm{H}, J=7.6 \mathrm{~Hz}, 1.2 \mathrm{~Hz})$, 7.59-7.54 (m, 2H), $7.50(\mathrm{td}, 1 \mathrm{H}, J=7.5,1.0 \mathrm{~Hz}), 7.39-7.34(\mathrm{~m}, 2 \mathrm{H}), 7.34-7.27(\mathrm{~m}, 1 \mathrm{H}), 6.70(\mathrm{~s}, 1 \mathrm{H})$, 3.47 (s, 3H). ${ }^{13} \mathrm{C} \mathrm{NMR}\left(\mathrm{CDCl}_{3}, 101 \mathrm{MHz}\right): \delta 162.8,134.6,133.2,132.5,130.3,129.5,129.3,127.9,127.8$, 126.0, 123.5, 119.5, 107.0, 63.4. HRMS (ESI-TOF) m/z: $[\mathrm{M}+\mathrm{H}]^{+}$Calcd for $\mathrm{C}_{16} \mathrm{H}_{14} \mathrm{NO}_{2} 252.1024$; Found: 252.1046; [M+H+CH$\left.{ }_{3} \mathrm{CN}\right]^{+}$Calcd for $\mathrm{C}_{18} \mathrm{H}_{17} \mathrm{~N}_{2} \mathrm{O}_{2} 293.1290$; Found: 293.1334.

(E)-3-Benzylidene-2-methoxyisoindolin-1-one, $\boldsymbol{E}$-2aa. Previously reported either as a slightly yellow oil $^{9}$ or a pale yellow solid. ${ }^{10}$ In this work, it is isolated as a colorless solid (30 mg, $0.12 \mathrm{mmol}, 40 \%$ ), $\mathrm{mp}$ 133-134 ${ }^{\circ} \mathrm{C} .{ }^{1} \mathrm{H}$ NMR $\left(\mathrm{CDCl}_{3}, 400 \mathrm{MHz}\right): \delta 7.86-7.81(\mathrm{~m}, 1 \mathrm{H}), 7.50-7.34(\mathrm{~m}, 8 \mathrm{H}), 6.76(\mathrm{~s}, 1 \mathrm{H}), 4.10(\mathrm{~s}$, 3H). ${ }^{13} \mathrm{C} \mathrm{NMR}\left(\mathrm{CDCl}_{3}, 101 \mathrm{MHz}\right): \delta 161.0,134.5,132.0,131.8,131.4,129.6,129.5,128.8,128.1,127.9$, 123.4, 123.0, 110.1, 64.3. HRMS (ESI-TOF) $\mathrm{m} / \mathrm{z}$ : $[\mathrm{M}+\mathrm{H}]^{+}$Calcd for $\mathrm{C}_{16} \mathrm{H}_{14} \mathrm{NO}_{2} 252.1024$; Found: 252.1029; $\left[\mathrm{M}+\mathrm{H}+\mathrm{CH}_{3} \mathrm{CN}\right]^{+}$Calcd for $\mathrm{C}_{18} \mathrm{H}_{17} \mathrm{~N}_{2} \mathrm{O}_{2}$ 293.1290; Found: 293.1292.

(Z)-3-(4-Chlorobenzylidene)-2-methoxyisoindolin-1-one, $Z$-2ab. Light yellow solid (19 mg, 0.067 mmol, 22\%). ${ }^{1} \mathrm{H}$ NMR $\left(\mathrm{CDCl}_{3}, 400 \mathrm{MHz}\right): \delta 7.90-7.84(\mathrm{~m}, 1 \mathrm{H}), 7.74-7.71(\mathrm{~m}, 1 \mathrm{H}), 7.64(\mathrm{td}, 1 \mathrm{H}, J=7.6$, 1.2 Hz), 7.54-7.48 (m, 2H), 7.38-7.30 (m, 4H), $6.62(\mathrm{~s}, 1 \mathrm{H}), 3.51(\mathrm{~s}, 3 \mathrm{H})$. Insufficient quantity obtained for ${ }^{13} \mathrm{C}$ NMR spectroscopy. HRMS (ESI-TOF) m/z: $[\mathrm{M}+\mathrm{H}]^{+}$Calcd for $\mathrm{C}_{16} \mathrm{H}_{13} \mathrm{NO}_{2} \mathrm{Cl} 286.0634$; Found: 286.0635.

(E)-3-(4-Chlorobenzylidene)-2-methoxyisoindolin-1-one, $\boldsymbol{E}$-2ab. Light yellow solid (40 mg, 0.139 mmol, 46\%). mp 110-111 ${ }^{\circ} \mathrm{C} .{ }^{1} \mathrm{H} \mathrm{NMR}\left(\mathrm{CDCl}_{3}, 400 \mathrm{MHz}\right): \delta 7.86-7.83(\mathrm{~m}, 1 \mathrm{H}), 7.51-7.37(\mathrm{~m}, 7 \mathrm{H}), 6.67$ 
(s, 1H), $4.10(\mathrm{~s}, 3 \mathrm{H}) .{ }^{13} \mathrm{C} \mathrm{NMR}\left(\mathrm{CDCl}_{3}, 101 \mathrm{MHz}\right): \delta 161.1,134.0,133.0,132.4,132.2,131.2,130.9$, 129.9, 129.1, 128.0, 123.6, 122.9, 108.5, 64.4. HRMS (ESI-TOF) m/z: $[\mathrm{M}+\mathrm{H}]^{+}$Calcd for $\mathrm{C}_{16} \mathrm{NO}_{2} \mathrm{H}_{13} \mathrm{Cl}$ 286.0634; Found: 286.0630; [M+H+CH$\left.{ }_{3} \mathrm{CN}\right]^{+}$Calcd for $\mathrm{C}_{18} \mathrm{H}_{16} \mathrm{~N}_{2} \mathrm{O}_{2} \mathrm{Cl}$ 327.0900; Found: 327.0910 .

(Z)-3-(4-Chlorobenzylidene)-2-methoxy-5-methyl-isoindolin-1-one, $\boldsymbol{Z}$-2db. Light yellow solid (28 mg, $0.093 \mathrm{mmol}, 31 \%) .{ }^{1} \mathrm{H} \mathrm{NMR}\left(\mathrm{CDCl}_{3}, 400 \mathrm{MHz}\right): \delta 7.73(\mathrm{~d}, 1 \mathrm{H}, J=7.9 \mathrm{~Hz}), 7.53-7.46(\mathrm{~m}, 3 \mathrm{H})$, 7.35-7.30 (m, 3H), $6.58(\mathrm{~s}, 1 \mathrm{H}), 3.48(\mathrm{~s}, 3 \mathrm{H}), 2.50(\mathrm{~s}, 3 \mathrm{H}) .{ }^{13} \mathrm{C} \mathrm{NMR}\left(\mathrm{CDCl}_{3}, 101 \mathrm{MHz}\right): \delta 163.1,143.5$, $134.8,133.6,131.9,131.6,130.9,130.63,130.2,129.0,128.1,123.6,119.9,105.0,63.3,22.3$. HRMS (ESI-TOF) m/z: [M+H] ${ }^{+}$Calcd for $\mathrm{C}_{17} \mathrm{H}_{15} \mathrm{NO}_{2} \mathrm{Cl} 300.0791$; Found: 300.0800 ; $\left[\mathrm{M}+\mathrm{H}+\mathrm{CH}_{3} \mathrm{CN}\right]^{+} \mathrm{Calcd}$ for $\mathrm{C}_{19} \mathrm{H}_{18} \mathrm{~N}_{2} \mathrm{O}_{2} \mathrm{Cl}$ 341.1056; Found: 341.1052.

(E)-3-(4-Chlorobenzylidene)-2-methoxy-5-methyl-isoindolin-1-one, $\boldsymbol{E}-2 \mathrm{db}$. Light yellow solid (36 mg, $0.120 \mathrm{mmol}, 40 \%) . \mathrm{mp} 137-139{ }^{\circ} \mathrm{C} .{ }^{1} \mathrm{H} \mathrm{NMR}\left(\mathrm{CDCl}_{3}, 400 \mathrm{MHz}\right): \delta 7.73(\mathrm{dd}, 1 \mathrm{H}, J=7.5,0.9 \mathrm{~Hz})$, 7.45-7.38 (m, 4H), 7.29-7.26 (m, 2H), $6.62(\mathrm{~s}, 1 \mathrm{H}), 4.08(\mathrm{~s}, 3 \mathrm{H}), 2.31(\mathrm{~s}, 3 \mathrm{H}) .{ }^{13} \mathrm{C} \mathrm{NMR}\left(\mathrm{CDCl}_{3}, 101\right.$ MHz): $\delta 161.3,143.0,133.9,133.1,132.5,131.5,130.9,129.9,128.1,125.4,123.5,123.4,123.3,108.2$, 22.3. HRMS (ESI-TOF) m/z: $[\mathrm{M}+\mathrm{H}]^{+}$Calcd for $\mathrm{C}_{17} \mathrm{H}_{15} \mathrm{NO}_{2} \mathrm{Cl}$ 300.0791; Found: 300.0779; $\left[\mathrm{M}+\mathrm{H}+\mathrm{CH}_{3} \mathrm{CN}\right]^{+}$Calcd for $\mathrm{C}_{19} \mathrm{H}_{18} \mathrm{~N}_{2} \mathrm{O}_{2} \mathrm{Cl} 341.1056$; Found: 341.1057.

(Z)-3-(4-Chlorobenzylidene)-2-methoxy-6-methyl-isoindolin-1-one, $Z$-2gb. Light yellow solid (13 mg, $0.043 \mathrm{mmol}, 15 \%) .{ }^{1} \mathrm{H} \mathrm{NMR}\left(\mathrm{CDCl}_{3}, 400 \mathrm{MHz}\right): \delta 7.67-7.68(\mathrm{~m}, 1 \mathrm{H}), 7.62-7.59(\mathrm{~m}, 1 \mathrm{H}), 7.51-7.47$ $(\mathrm{m}, 2 \mathrm{H}), 7.45-7.42(\mathrm{~m}, 1 \mathrm{H}), 7.35-7.30(\mathrm{~m}, 2 \mathrm{H}), 6.55(\mathrm{~s}, 1 \mathrm{H}), 3.49(\mathrm{~s}, 3 \mathrm{H}), 2.47(\mathrm{~s}, 3 \mathrm{H}) .{ }^{13} \mathrm{C} \mathrm{NMR}\left(\mathrm{CDCl}_{3}\right.$, $101 \mathrm{MHz}): \delta 163.1,140.1,133.7,133.6,131.9,131.6,130.2,128.1,126.1,123.8,119.4,104.8,63.3$, 21.7. HRMS (ESI-TOF) $\mathrm{m} / \mathrm{z}: \quad[\mathrm{M}+\mathrm{H}]^{+}$Calcd for $\mathrm{C}_{17} \mathrm{H}_{15} \mathrm{NO}_{2} \mathrm{Cl}$ 300.0791; Found: 300.0800; $\left[\mathrm{M}+\mathrm{H}+\mathrm{CH}_{3} \mathrm{CN}\right]^{+}$Calcd for $\mathrm{C}_{19} \mathrm{H}_{18} \mathrm{~N}_{2} \mathrm{O}_{2} \mathrm{Cl} 341.1056$; Found: 341.1055 . 
(E)-3-(4-Chlorobenzylidene)-2-methoxy-6-methyl-isoindolin-1-one, $\boldsymbol{E}$-2gb. Light yellow solid (42 mg, 0.14 mmol, 47\%). mp 141-142 ${ }^{\circ} \mathrm{C} .{ }^{1} \mathrm{H}$ NMR $\left(\mathrm{CDCl}_{3}, 400 \mathrm{MHz}\right): \delta 7.65(\mathrm{~d}, 1 \mathrm{H}, J=2.7 \mathrm{~Hz}), 7.45-$ $7.32(\mathrm{~m}, 5 \mathrm{H}), 7.20(\mathrm{dd}, 1 \mathrm{H}, J=8.1,1.8 \mathrm{~Hz}), 6.61(\mathrm{~s}, 1 \mathrm{H}), 4.09(\mathrm{~s}, 3 \mathrm{H}), 2.41(\mathrm{~s}, 3 \mathrm{H}) .{ }^{13} \mathrm{C} \mathrm{NMR}\left(\mathrm{CDCl}_{3}\right.$, $101 \mathrm{MHz}): \delta 161.3,140.5,133.8,133.2,133.1,132.5,130.9,129.0,128.6,128.1,123.8,122.8,107.6$, 64.3, 21.7. HRMS (ESI-TOF) $\mathrm{m} / \mathrm{z}$ : $[\mathrm{M}+\mathrm{H}]^{+}$Calcd for $\mathrm{C}_{17} \mathrm{NO}_{2} \mathrm{H}_{15} \mathrm{Cl}$ 300.0791; Found: 300.0807; $\left[\mathrm{M}+\mathrm{H}+\mathrm{CH}_{3} \mathrm{CN}\right]^{+}$Calcd for $\mathrm{C}_{19} \mathrm{H}_{18} \mathrm{~N}_{2} \mathrm{O}_{2} \mathrm{Cl} 341.1056$; Found: 341.1083 .

(Z)-3-(4-Chlorobenzylidene)-2,6-dimethoxy-isoindolin-1-one, $Z$-2hb. Light yellow solid (14 mg, $0.044 \mathrm{mmol}, 15 \%) .{ }^{1} \mathrm{H} \mathrm{NMR}\left(\mathrm{CDCl}_{3}, 400 \mathrm{MHz}\right): \delta 7.63-7.59(\mathrm{~m}, 1 \mathrm{H}), 7.50-7.46(\mathrm{~m}, 2 \mathrm{H}), 7.35-7.30(\mathrm{~m}$, 3H), $7.17(\mathrm{dd}, 1 \mathrm{H}, J=8.5,2.5 \mathrm{~Hz}), 6.50(\mathrm{~s}, 1 \mathrm{H}), 3.89(\mathrm{~s}, 3 \mathrm{H}), 3.49(\mathrm{~s}, 3 \mathrm{H}) .{ }^{13} \mathrm{C} \mathrm{NMR}\left(\mathrm{CDCl}_{3}, 101\right.$ MHz): $\delta 163.0,161.2,133.5,131.9,131.6,130.0,128.1,127.5,127.0,121.1,121.0,106.3,104.5,63.4$, 56.0. HRMS (ESI-TOF) m/z: $[\mathrm{M}+\mathrm{H}]^{+}$Calcd for $\mathrm{C}_{17} \mathrm{H}_{15} \mathrm{NO}_{3} \mathrm{Cl}$ 316.0740; Found: 316.0727; $\left[\mathrm{M}+\mathrm{H}+\mathrm{CH}_{3} \mathrm{CN}\right]^{+}$Calcd for $\mathrm{C}_{19} \mathrm{H}_{18} \mathrm{~N}_{2} \mathrm{O}_{3} \mathrm{Cl}$ 357.1006; Found: 357.0928.

(E)-3-(4-Chlorobenzylidene)-2,6-dimethoxy-isoindolin-1-one, $\boldsymbol{E}$-2hb. Light yellow solid (45 mg, $0.145 \mathrm{mmol}, 49 \%) . \mathrm{mp} 109-110{ }^{\circ} \mathrm{C} .{ }^{1} \mathrm{H} \mathrm{NMR}\left(\mathrm{CDCl}_{3}, 400 \mathrm{MHz}\right): \delta 7.63-7.59(\mathrm{~m}, 1 \mathrm{H}), 7.50-7.46(\mathrm{~m}$, 2H), 7.35-7.30 (m, 3H), $7.17(\mathrm{dd}, 1 \mathrm{H}, J=8.5,2.5 \mathrm{~Hz}), 6.50(\mathrm{~s}, 1 \mathrm{H}), 3.89(\mathrm{~s}, 3 \mathrm{H}), 3.49(\mathrm{~s}, 3 \mathrm{H}) .{ }^{13} \mathrm{C} \mathrm{NMR}$ $\left(\mathrm{CDCl}_{3}, 101 \mathrm{MHz}\right): \delta 161.2,161.1,133.7,133.2,132.3,130.8,129.7,129.9,124.3,123.7,119.9,107.0$ 106.6, 64.4, 55.8. HRMS (ESI-TOF) m/z: $[\mathrm{M}+\mathrm{H}]^{+}$Calcd for $\mathrm{C}_{17} \mathrm{H}_{15} \mathrm{NO}_{3} \mathrm{Cl}$ 316.0740; Found: 316.0735 ; $\left[\mathrm{M}+\mathrm{H}+\mathrm{CH}_{3} \mathrm{CN}\right]^{+}$Calcd for $\mathrm{C}_{19} \mathrm{H}_{18} \mathrm{~N}_{2} \mathrm{O}_{3} \mathrm{Cl}$ 357.1006; Found: 357.1006 .

(Z)-3-(4-Chlorobenzylidene)-2,5-dimethoxy-isoindolin-1-one, Z-2ib. Light yellow solid (16 mg, 0.051 mmol, 17\%). mp 140-141 ${ }^{\circ} \mathrm{C} .{ }^{1} \mathrm{H}$ NMR $\left(\mathrm{CDCl}_{3}, 400 \mathrm{MHz}\right): \delta 7.80-7.75(\mathrm{~m}, 1 \mathrm{H}), 7.53-7.47(\mathrm{~m}, 2 \mathrm{H})$, 7.36-7.31 (m, 2H), 7.18-7.16 (m, 1H), $7.04(\mathrm{dd}, 1 \mathrm{H}, J=8.4,2.2 \mathrm{~Hz}), 6.56(\mathrm{~s}, 1 \mathrm{H}), 3.93(\mathrm{~s}, 3 \mathrm{H}), 3.48$ (s, 3H). ${ }^{13} \mathrm{C} \mathrm{NMR}\left(\mathrm{CDCl}_{3}, 101 \mathrm{MHz}\right): \delta 163.8,163.1,136.8,133.7,131.8,131.6,130.3,128.2,125.4,118.7$, 
116.5, 108.9, 105.1, 104.2, 63.2, 56.0. HRMS (ESI-TOF) m/z: $[\mathrm{M}+\mathrm{H}]^{+}$Calcd for $\mathrm{C}_{17} \mathrm{H}_{15} \mathrm{NO}_{3} \mathrm{Cl}$ 316.0740;

Found: 316.0725; [M+H+CH$\left.{ }_{3} \mathrm{CN}\right]^{+}$Calcd for $\mathrm{C}_{19} \mathrm{H}_{18} \mathrm{~N}_{2} \mathrm{O}_{3} \mathrm{Cl} 357.1006$; Found: 357.0992 .

(E)-3-(4-Chlorobenzylidene)-2,5-dimethoxy-isoindolin-1-one, $\boldsymbol{E}$-2ib. Light yellow solid (30 mg, 0.095 mmol, 32\%). mp 151-153 ${ }^{\circ} \mathrm{C} .{ }^{1} \mathrm{H}$ NMR $\left(\mathrm{CDCl}_{3}, 400 \mathrm{MHz}\right): \delta 7.79-7.73(\mathrm{~m}, 1 \mathrm{H}), 7.45-7.38(\mathrm{~m}, 4 \mathrm{H})$, 7.01-6.92 (m, 2H), $6.63(\mathrm{~s}, 1 \mathrm{H}), 4.08(\mathrm{~s}, 3 \mathrm{H}), 3.69(\mathrm{~s}, 3 \mathrm{H}) .{ }^{13} \mathrm{C} \mathrm{NMR}\left(\mathrm{CDCl}_{3}, 101 \mathrm{MHz}\right): \delta 163.1,161.4$, 134.0, 133.3, 133.1,132.7, 131.6, 130.9, 129.0, 128.1, 125.2, 120.5, 116.1, 108.3, 108.2, 64.3, 55.6. HRMS (ESI-TOF) m/z: $[\mathrm{M}+\mathrm{H}]^{+}$Calcd for $\mathrm{C}_{17} \mathrm{H}_{15} \mathrm{NO}_{3} \mathrm{Cl}$ 316.0740; Found: 316.0727 ; $\left[\mathrm{M}+\mathrm{H}+\mathrm{CH} \mathrm{CN}^{+}\right.$ Calcd for $\mathrm{C}_{19} \mathrm{H}_{18} \mathrm{~N}_{2} \mathrm{O}_{3} \mathrm{Cl}$ 357.1006; Found: 357.0990.

(E)-3-(4-Chlorobenzylidene)-5-trifluoromethyl-2-methoxy-isoindolin-1-one, $\boldsymbol{E}-2 \mathrm{jb}$. Colorless solid (18 mg, $0.051 \mathrm{mmol}, 17 \%) . \mathrm{mp} 147-149{ }^{\circ} \mathrm{C} .{ }^{1} \mathrm{H}$ NMR $\left(\mathrm{CDCl}_{3}, 400 \mathrm{MHz}\right): \delta 7.99-7.97$ (m, 1H), $7.79-$ $7.71(\mathrm{~m}, 2 \mathrm{H}), 7.48-7.40(\mathrm{~m}, 4 \mathrm{H}), 6.80(\mathrm{~s}, 1 \mathrm{H}), 4.13(\mathrm{~s}, 3 \mathrm{H}) .{ }^{13} \mathrm{C} \mathrm{NMR}\left(\mathrm{CDCl}_{3}, 101 \mathrm{MHz}\right): \delta 159.6,134.8$, $134.3,133.9,132.2,131.5,131.3,130.9,130.7,129.3,126.8,126.8,126.8,126.7,124.2,120.1,120.1$, 120.0, 120.0, 110.5, 64.6. HRMS (ESI-TOF) m/z: $[\mathrm{M}+\mathrm{H}]^{+}$Calcd for $\mathrm{C}_{17} \mathrm{H}_{12} \mathrm{NO}_{2} \mathrm{ClF}_{3}$ 354.0508; Found: 354.0501; [M+H+CH$\left.{ }_{3} \mathrm{CN}\right]^{+}$Calcd for $\mathrm{C}_{19} \mathrm{H}_{15} \mathrm{~N}_{2} \mathrm{O}_{2} \mathrm{ClF}_{3}$ 395.0774; Found: 395.0730.

3-Benzyl-3-hydro-2-methoxy-isoindolin-1-one, 4aa. Colorless solid (24 mg, $0.094 \mathrm{mmol}, 31 \%$ ). mp 94-95 ${ }^{\circ} \mathrm{C} .{ }^{1} \mathrm{H} \mathrm{NMR}\left(\mathrm{CDCl}_{3}, 400 \mathrm{MHz}\right): \delta$ 7.75-7.72 (m, 1H), 7.47-7.36 (m, 2H), 7.26-7.18 (m, 3H), 7.13-7.08 (m, 2H), 6.99-6.96 (m, 1H), $4.98(\mathrm{dd}, 1 \mathrm{H}, J=7.6,4.4 \mathrm{~Hz}), 3.99(\mathrm{~s}, 3 \mathrm{H}), 3.45(\mathrm{dd}, 1 \mathrm{H}, J=13.8$, 4.4 Hz), $3.02(\mathrm{dd}, 1 \mathrm{H}, J=13.8,7.6 \mathrm{~Hz}) .{ }^{13} \mathrm{C} \mathrm{NMR}\left(\mathrm{CDCl}_{3}, 101 \mathrm{MHz}\right): \delta 164.6,141.6,135.5,131.7,130.1$, 129.8, 128.5, 128.5, 127.1, 123.8, 123.1, 63.9, 60.4, 37.4. HRMS (ESI-TOF) m/z: $[\mathrm{M}+\mathrm{H}]^{+}$Calcd for $\mathrm{C}_{16} \mathrm{H}_{16} \mathrm{NO}_{2} 254.1181$; Found: 254.1181.

3-Benzyl-2,3-dihydro-isoindolin-1-one, 4'aa. Colorless solid (11 mg, 0.049 mmol, 16\%). mp 134-135 ${ }^{\circ} \mathrm{C} .{ }^{1} \mathrm{H} \mathrm{NMR}\left(\mathrm{CDCl}_{3}, 400 \mathrm{MHz}\right): \delta 7.83(\mathrm{dt}, 1 \mathrm{H}, J=7.3,1.0 \mathrm{~Hz}), 7.53(\mathrm{td}, 1 \mathrm{H}, J=7.5,1.3 \mathrm{~Hz}), 7.46(\mathrm{td}$, 
$1 \mathrm{H}, J=7.4,1.0 \mathrm{~Hz}), 7.34-7.28(\mathrm{~m}, 3 \mathrm{H}, \mathrm{Ar}-\mathrm{H}), 7.27(\mathrm{~d}, 2 \mathrm{H}, J=6.6 \mathrm{~Hz}), 7.20(\mathrm{~d}, 1 \mathrm{H}, J=1.4 \mathrm{~Hz}), 7.08(\mathrm{br}$ s, $1 \mathrm{H}, \mathrm{NH}), 4.81(\mathrm{dd}, 1 \mathrm{H}, J=8.5,5.5 \mathrm{~Hz}), 3.18(\mathrm{dd}, 1 \mathrm{H}, J=13.6,5.5 \mathrm{~Hz}), 2.87(\mathrm{dd}, 1 \mathrm{H}, J=13.6,8.5$ $\mathrm{Hz}) \cdot{ }^{13} \mathrm{C} \mathrm{NMR}\left(\mathrm{CDCl}_{3}, 101 \mathrm{MHz}\right): \delta 170.6,146.9,137.0,132.1,131.8,129.4,128.9,128.4,127.2,123.9$, 122.8, 58.1, 41.4. HRMS (ESI-TOF) m/z: $[\mathrm{M}+\mathrm{H}]^{+}$Calcd for $\mathrm{C}_{15} \mathrm{H}_{14} \mathrm{NO}$ 224.1075; Found: 224.1079; $\left[\mathrm{M}+\mathrm{H}+\mathrm{CH}_{3} \mathrm{CN}\right]^{+}$Calcd for $\mathrm{C}_{17} \mathrm{H}_{17} \mathrm{~N}_{2} \mathrm{O}$ 265.1341; Found: 265.1236 .

3-(4-Chlorobenzyl)-3-hydro-2-methoxy-isoindolin-1-one, 4ab. Colorless solid (22 mg, 0.076 mmol, 25\%). ${ }^{1} \mathrm{H}$ NMR $\left(\mathrm{CDCl}_{3}, 400 \mathrm{MHz}\right): \delta 7.72-7.69(\mathrm{~m}, 1 \mathrm{H}), 7.47$ (td, 1H, $\left.J=7.5,1.3 \mathrm{~Hz}\right), 7.39(\mathrm{ddd}, 1 \mathrm{H}, J$ $=8.2,7.5,1.0 \mathrm{~Hz}), 7.18-7.06(\mathrm{~m}, 3 \mathrm{H}), 7.02-6.95(\mathrm{~m}, 2 \mathrm{H}), 4.97(\mathrm{dd}, 1 \mathrm{H}, J=6.8,4.2 \mathrm{~Hz}), 3.98(\mathrm{~s}, 3 \mathrm{H})$, $3.33(\mathrm{dd}, 1 \mathrm{H}, J=13.9,4.2 \mathrm{~Hz}), 3.11(\mathrm{dd}, 1 \mathrm{H}, J=13.9,6.8 \mathrm{~Hz}) .{ }^{13} \mathrm{C} \mathrm{NMR}\left(\mathrm{CDCl}_{3}, 101 \mathrm{MHz}\right): \delta 164.4$, $141.1,133.5,132.9,131.8,131.2,130.1,128.7,128.5,123.9,122.9,63.8,59.8,36.8$. HRMS (ESI-TOF) $\mathrm{m} / \mathrm{z}: \quad[\mathrm{M}+\mathrm{H}]^{+}$Calcd for $\mathrm{C}_{16} \mathrm{H}_{15} \mathrm{NO}_{2} \mathrm{Cl}$ 288.0791; Found: 288.0782; $\left[\mathrm{M}+\mathrm{H}+\mathrm{CH}_{3} \mathrm{CN}\right]^{+} \mathrm{Calcd}$ for $\mathrm{C}_{18} \mathrm{H}_{18} \mathrm{~N}_{2} \mathrm{O}_{2} \mathrm{Cl}$ 329.1057; Found: 329.1043.

3-(4-Fluorobenzyl)-3-hydro-2-methoxy-isoindolin-1-one, 4ac. Colorless solid (10 mg, $0.036 \mathrm{mmol}$, 12\%). ${ }^{1} \mathrm{H} \mathrm{NMR}\left(\mathrm{CDCl}_{3}, 400 \mathrm{MHz}\right): \delta 7.72(\mathrm{dt}, 1 \mathrm{H}, J=7.5,1.0 \mathrm{~Hz}), 7.47(\mathrm{td}, 1 \mathrm{H}, J=7.5,1.3 \mathrm{~Hz}), 7.40-$ $7.37(\mathrm{~m}, 1 \mathrm{H}), 7.09-7.06(\mathrm{~m}, 1 \mathrm{H}), 7.02-6.99(\mathrm{~m}, 2 \mathrm{H}),, 6.90-6.84(\mathrm{~m}, 2 \mathrm{H}), 4.97$ (dd, 1H, J=6.8, 4.2 Hz), $3.99(\mathrm{~s}, 3 \mathrm{H}), 3.35(\mathrm{dd}, 1 \mathrm{H}, J=14.0,4.2 \mathrm{~Hz}), 3.12(\mathrm{dd}, 1 \mathrm{H}, J=14.0,6.8 \mathrm{~Hz}) .{ }^{13} \mathrm{C} \mathrm{NMR}\left(\mathrm{CDCl}_{3}, 101 \mathrm{MHz}\right)$ : $\delta 164.5,162.0(\mathrm{~d}, J=254 \mathrm{~Hz}), 141.3,131.6(\mathrm{~d}, J=8.0 \mathrm{~Hz}), 131.3,130.7,130.2,128.7,123.9,122.9$, $115.3(\mathrm{~d}, J=22 \mathrm{~Hz}), 63.8,60.0,36.7$. HRMS (ESI-TOF) $\mathrm{m} / \mathrm{z}:[\mathrm{M}+\mathrm{H}]^{+}$Calcd for $\mathrm{C}_{16} \mathrm{H}_{15} \mathrm{NO}_{2} \mathrm{~F} 272.1086$; Found: 272.1086; [M+H+CH$\left.{ }_{3} \mathrm{CN}\right]^{+}$Calcd for $\mathrm{C}_{18} \mathrm{H}_{18} \mathrm{~N}_{2} \mathrm{O}_{2} \mathrm{~F}$ 313.1352; Found: 313.1275 .

3-(4-Fluorobenzyl)-2,3-dihydro-isoindolin-1-one, 4'ac. Colorless solid (18 mg, $0.075 \mathrm{mmol}, 25 \%$ ). mp 147-149 ${ }^{\circ} \mathrm{C} .{ }^{1} \mathrm{H}$ NMR $\left(\mathrm{CDCl}_{3}, 400 \mathrm{MHz}\right): \delta 7.81(\mathrm{dt}, 1 \mathrm{H}, J=7.5,1.0 \mathrm{~Hz}, \mathrm{Ar}-\mathrm{H}), 7.54(\mathrm{td}, 1 \mathrm{H}, J=7.5,1.3$ Hz, Ar-H), 7.50-7.43 (m, 1H, F Ar-H), 7.38 (d, 1H, J=6.0 Hz, Ar-H), 7.29-7.26 (m, 1H), 7.17-7.10 (m, 2H), 7.01-6.92 (m, 2H), 4.97-4.67 (m, 1H), $3.15(\mathrm{dd}, 1 \mathrm{H}, J=13.7,5.6 \mathrm{~Hz}), 2.90(\mathrm{dd}, 1 \mathrm{H}, J=13.7,7.9$ 
Hz). ${ }^{13} \mathrm{C} \mathrm{NMR}\left(\mathrm{CDCl}_{3}, 101 \mathrm{MHz}\right): \delta 170.9,162.0(\mathrm{~d}, J=245 \mathrm{~Hz}), 146.7,132.4(\mathrm{~d}, J=3.2 \mathrm{~Hz}), 132.1$, 131.8, $131.0(\mathrm{~d}, J=8.1 \mathrm{~Hz}), 128.5,124.0,122.8,115.6(\mathrm{~d}, J=21.6 \mathrm{~Hz}), 58.0,40.4$. HRMS (ESI-TOF) m/z: $[\mathrm{M}+\mathrm{H}]^{+}$Calcd for $\mathrm{C}_{15} \mathrm{H}_{13} \mathrm{NOF} 242.0981$; Found: 242.0983 ; $\left[\mathrm{M}+\mathrm{H}+\mathrm{CH}_{3} \mathrm{CN}\right]^{+} \mathrm{Calcd}$ for $\mathrm{C}_{17} \mathrm{H}_{16} \mathrm{~N}_{2} \mathrm{OF}$ 283.1247; Found: 283.1242.

3-Hydro-2-methoxy-3-(4-methylbenzyl)-isoindolin-1-one, 4ad. Colorless solid (26 mg, $0.097 \mathrm{mmol}$, $32 \%) .{ }^{1} \mathrm{H} \mathrm{NMR}\left(\mathrm{CDCl}_{3}, 400 \mathrm{MHz}\right): \delta 7.73(\mathrm{ddd}, 1 \mathrm{H}, J=7.3,1.5,0.8 \mathrm{~Hz}), 7.47-7.34(\mathrm{~m}, 2 \mathrm{H}), 7.05-6.95$ (m, 5H), $4.95(\mathrm{dd}, 1 \mathrm{H}, J=7.7,4.3 \mathrm{~Hz}), 3.98(\mathrm{~s}, 3 \mathrm{H}), 3.41(\mathrm{dd}, 1 \mathrm{H}, J=13.8,4.3 \mathrm{~Hz}), 2.96(\mathrm{dd}, 1 \mathrm{H}, J=$ 13.8, $7.7 \mathrm{~Hz}), 2.28(\mathrm{~s}, 3 \mathrm{H}) .{ }^{13} \mathrm{C} \mathrm{NMR}\left(\mathrm{CDCl}_{3}, 101 \mathrm{MHz}\right): \delta 164.5,141.7,136.6,132.2,131.7,130.0$, 129.6, 129.1, 128.4, 123.7, 123.1, 63.9, 60.4, 37.3, 21.2. HRMS (ESI-TOF) m/z: [M+H] Calcd for $\mathrm{C}_{17} \mathrm{H}_{18} \mathrm{NO}_{2} 268.1337$; Found: 268.1328 .

2,3-Dihydro-3-(4-methylbenzyl)-isoindolin-1-one, 4'ad. Colorless solid (4 mg, $0.017 \mathrm{mmol}, 6 \%) .{ }^{1} \mathrm{H}$ NMR $\left(\mathrm{CDCl}_{3}, 400 \mathrm{MHz}\right): \delta 7.85-7.82(\mathrm{~m}, 1 \mathrm{H}), 7.56(\mathrm{td}, 1 \mathrm{H}, J=7.5,1.2 \mathrm{~Hz}), 7.49-7.46(\mathrm{~m}, 1 \mathrm{H}), 7.37-$ $7.34(\mathrm{~m}, 1 \mathrm{H}), 7.18-7.10(\mathrm{~m}, 4 \mathrm{H}), 6.33$ (br s, 1H), $4.76(\mathrm{dd}, 1 \mathrm{H}, J=9.4,4.9 \mathrm{~Hz}), 3.22(\mathrm{dd}, 1 \mathrm{H}, J=13.6$, $4.9 \mathrm{~Hz}), 2.72(\mathrm{dd}, 1 \mathrm{H}, J=13.6,9.4 \mathrm{~Hz}), 2.35(\mathrm{~s}, 3 \mathrm{H}) .{ }^{13} \mathrm{C} \mathrm{NMR}\left(\mathrm{CDCl}_{3}, 101 \mathrm{MHz}\right): \delta 170.4,147.0,137.0$, 134.0, 131.9, 129.7, 129.2, 128.5, 124.1, 122.8, 58.3, 41.2, 21.2. HRMS (ESI-TOF) m/z: $[\mathrm{M}+\mathrm{H}]^{+}$Calcd for $\mathrm{C}_{16} \mathrm{H}_{16} \mathrm{NO} 238.1231$; Found: 238.1237; $\left[\mathrm{M}+\mathrm{H}+\mathrm{CH}_{3} \mathrm{CN}\right]^{+}$Calcd for $\mathrm{C}_{18} \mathrm{H}_{19} \mathrm{~N}_{2} \mathrm{O}$ 279.1497; Found: 279.1386.

3-Hydro-2-methoxy-3-(2-naphthylmethyl)-isoindolin-1-one, 4ae. Colorless solid (27 mg, 0.088 mmol, 29 \%). ${ }^{1} \mathrm{H} \mathrm{NMR}\left(\mathrm{CDCl}_{3}, 400 \mathrm{MHz}\right): \delta 7.83-7.77$ (m, 1H), 7.76-7.71 (m, 3H), 7.60-7.56 (m, 1H), 7.48$7.40(\mathrm{~m}, 2 \mathrm{H}), 7.40-7.34(\mathrm{~m}, 2 \mathrm{H}), 7.31-7.23(\mathrm{~m}, 1 \mathrm{H}), 6.98-6.94(\mathrm{~m}, 1 \mathrm{H}), 5.07$ (dd,1H, J =7.8, $4.4 \mathrm{~Hz})$, $4.01(\mathrm{~s}, 3 \mathrm{H}), 3.63(\mathrm{dd}, 1 \mathrm{H}, J=13.9,7.8 \mathrm{~Hz}), 3.14(\mathrm{dd}, 1 \mathrm{H}, J=13.8,7.8 \mathrm{~Hz}) .{ }^{13} \mathrm{C} \mathrm{NMR}\left(\mathrm{CDCl}_{3}, 101 \mathrm{MHz}\right)$ : $\delta 164.6,141.6,133.4,133.1,132.5,131.8,130.0,128.6,128.6,128.2,127.8,126.2,125.8,123.8,123.2$, 63.9, 60.3, 38.8. HRMS (ESI-TOF) m/z: [M+H] ${ }^{+}$Calcd for $\mathrm{C}_{20} \mathrm{H}_{18} \mathrm{NO}_{2}$ 304.1337; Found: 304.1330. 
2,3-Dihydro-3-(2-naphthylmethyl)-isoindolin-1-one, 4'ae. Colorless solid (12 mg, $0.043 \mathrm{mmol}, 14 \%$ ).

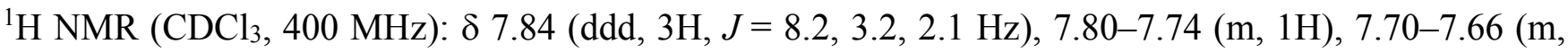
1H), 7.59-7.45 (m, 4H), 7.37 (ddd, $2 \mathrm{H}, J=7.5,4.5,1.4 \mathrm{~Hz}), 6.73$ (br s, $1 \mathrm{H}), 4.89$ (dd, $1 \mathrm{H}, J=9.1,5.1$ $\mathrm{Hz}), 3.38(\mathrm{dd}, 1 \mathrm{H}, J=13.6,5.1 \mathrm{~Hz}), 2.95(\mathrm{dd}, 1 \mathrm{H}, J=13.6,9.1 \mathrm{~Hz}) .{ }^{13} \mathrm{C} \mathrm{NMR}\left(\mathrm{CDCl}_{3}, 101 \mathrm{MHz}\right): \delta$ $170.5,147.0,134.6,133.6,132.6,132.0,131.9,128.8,128.5,128.1,127.8,127.7,127.2,126.5,126.0$, 124.1, 122.8, 58.1, 41.8. HRMS (ESI-TOF) m/z: $[\mathrm{M}+\mathrm{H}]^{+}$Calcd for $\mathrm{C}_{19} \mathrm{H}_{16} \mathrm{NO} 274.1231$; Found: 274.1242; $\left[\mathrm{M}+\mathrm{H}+\mathrm{CH}_{3} \mathrm{CN}\right]^{+}$Calcd for $\mathrm{C}_{21} \mathrm{H}_{19} \mathrm{~N}_{2} \mathrm{O} 315.1497$; Found: 315.1387.

3-(4-Trifluoromethyl-benzyl)-3-hydro-2-methoxy-isoindolin-1-one, 4af. Colorless solid (8 mg, 0.024 mmol, 8\%). ${ }^{1} \mathrm{H}$ NMR $\left(\mathrm{CDCl}_{3}, 400 \mathrm{MHz}\right): \delta 7.74-7.71(\mathrm{~m}, 1 \mathrm{H}), 7.52-7.38(\mathrm{~m}, 4 \mathrm{H}), 7.21-7.17(\mathrm{~m}, 2 \mathrm{H})$, 7.12-7.09 (m, 1H), $5.03(\mathrm{dd}, 1 \mathrm{H}, J=6.7,4.2 \mathrm{~Hz}), 3.99$ (s, 3H), $3.42(\mathrm{dd}, 1 \mathrm{H}, J=13.9,4.2 \mathrm{~Hz}), 3.22(\mathrm{dd}$, $1 \mathrm{H}, J=13.9,6.7 \mathrm{~Hz}) .{ }^{13} \mathrm{C} \mathrm{NMR}\left(\mathrm{CDCl}_{3}, 101 \mathrm{MHz}\right): \delta 164.6,141.0,139.3,132.0,130.2,130.1,128.9$, $125.3(\mathrm{q}, J=3.7 \mathrm{~Hz}), 124.0,122.8,63.9,59.7,37.3$. HRMS (ESI-TOF) m/z: $[\mathrm{M}+\mathrm{H}]^{+}$Calcd for $\mathrm{C}_{17} \mathrm{H}_{15} \mathrm{NO}_{2} \mathrm{~F}_{3}$ 322.1054; Found: 322.1056; $\left[\mathrm{M}+\mathrm{H}+\mathrm{CH}_{3} \mathrm{CN}\right]^{+}$Calcd for $\mathrm{C}_{19} \mathrm{H}_{18} \mathrm{~N}_{2} \mathrm{O}_{2} \mathrm{~F}_{3}$ 363.1320; Found: 363.1281.

3-(4-Trifluoromethylbenzyl)-2,3-dihydro-isoindolin-1-one, 4'af. Colorless solid (46 mg, 0.159 mmol, 53\%). mp 167-168 ${ }^{\circ} \mathrm{C} .{ }^{1} \mathrm{H}$ NMR $\left(\mathrm{CDCl}_{3}, 400 \mathrm{MHz}\right): \delta 7.85-7.71(\mathrm{~m}, 2 \mathrm{H}), 7.59-7.45(\mathrm{~m}, 4 \mathrm{H}), 7.30(\mathrm{dd}$, $3 \mathrm{H}, J=7.8,6.2 \mathrm{~Hz}), 4.88-4.84(\mathrm{~m}, 1 \mathrm{H}), 3.25(\mathrm{dd}, 1 \mathrm{H}, J=13.7,5.7 \mathrm{~Hz}), 3.02(\mathrm{dd}, 1 \mathrm{H}, J=13.7,7.6 \mathrm{~Hz})$. ${ }^{13} \mathrm{C} \mathrm{NMR}\left(\mathrm{CDCl}_{3}, 101 \mathrm{MHz}\right): \delta 171.0,146.5,140.8,132.1,132.0,129.9,129.6,129.3,128.7,125.6(\mathrm{q}$, $J=3.6 \mathrm{~Hz}), 124.0,122.8,57.7,40.9$. HRMS (ESI-TOF) m/z: $[\mathrm{M}+\mathrm{H}]^{+}$Calcd for $\mathrm{C}_{16} \mathrm{H}_{13} \mathrm{NOF}_{3}$ 292.0949; Found: 292.0950; [M+H+CH$\left.{ }_{3} \mathrm{CN}\right]^{+}$Calcd for $\mathrm{C}_{18} \mathrm{H}_{16} \mathrm{~N}_{2} \mathrm{OF}_{3} 333.1214$; Found: 333.1068 .

3-Benzyl-3-hydro-2-methoxy-5-methyl-isoindolin-1-one, 4da. Colorless solid (25 mg, $0.093 \mathrm{mmol}$, 31\%). ${ }^{1} \mathrm{H} \mathrm{NMR}\left(\mathrm{CDCl}_{3}, 400 \mathrm{MHz}\right): \delta 7.61(\mathrm{~d}, 1 \mathrm{H}, J=7.7 \mathrm{~Hz}), 7.25-7.15(\mathrm{~m}, 4 \mathrm{H}), 7.12-7.07(\mathrm{~m}, 2 \mathrm{H})$, 6.79-6.76 (m, 1H), $4.92(\mathrm{dd}, 1 \mathrm{H}, J=7.4,4.5 \mathrm{~Hz}), 3.94(\mathrm{~s}, 3 \mathrm{H}), 3.40(\mathrm{dd}, 1 \mathrm{H}, J=13.8,4.5 \mathrm{~Hz}), 3.01(\mathrm{dd}$, 
$1 \mathrm{H}, J=13.8,7.4 \mathrm{~Hz}), 2.34(\mathrm{~s}, 3 \mathrm{H}) .{ }^{13} \mathrm{C} \mathrm{NMR}\left(\mathrm{CDCl}_{3}, 101 \mathrm{MHz}\right): \delta 164.9,142.4,142.0,135.6,129.8$, 129.4, 128.4, 127.3, 127.0, 123.6, 63.8, 60.3, 37.8, 22.1. HRMS (ESI-TOF) m/z: [M+H] ${ }^{+}$Calcd for $\mathrm{C}_{17} \mathrm{H}_{18} \mathrm{NO}_{2}$ 268.1337; Found: 268.1324.

3-Benzyl-2,3-dihydro-5-methyl-isoindolin-1-one, 4'da. Colorless solid (15 mg, 0.065 mmol, 22\%). mp 147-148 ${ }^{\circ} \mathrm{C} .{ }^{1} \mathrm{H}$ NMR $\left(\mathrm{CDCl}_{3}, 400 \mathrm{MHz}\right): \delta 7.71(\mathrm{~d}, 1 \mathrm{H}, J=7.7 \mathrm{~Hz}), 7.37-7.26(\mathrm{~m}, 4 \mathrm{H}), 7.24-7.20$ (m, 2H), 7.13 (br s, 1H), 6.63-6.60 (m, 1H), $4.73(\mathrm{dd}, 1 \mathrm{H}, J=9.1,5.0 \mathrm{~Hz}), 3.22(\mathrm{dd}, 1 \mathrm{H}, J=13.6,5.0 \mathrm{~Hz})$, $2.76(\mathrm{dd}, 1 \mathrm{H}, J=13.6,9.1 \mathrm{~Hz}), 2.44(\mathrm{~s}, 3 \mathrm{H}) .{ }^{13} \mathrm{C} \mathrm{NMR}\left(\mathrm{CDCl}_{3}, 101 \mathrm{MHz}\right): \delta 170.0,147.0,142.6,137.2$, 129.5, 129.4, 129.3, 128.9, 127.2, 123.8, 123.3, 58.0, 41.6, 22.1. HRMS (ESI-TOF) m/z: [M+H] ${ }^{+}$Calcd for $\mathrm{C}_{16} \mathrm{H}_{16} \mathrm{NO} 238.1231$; Found: 238.1229; $\left[\mathrm{M}+\mathrm{H}+\mathrm{CH}_{3} \mathrm{CN}\right]^{+}$Calcd for $\mathrm{C}_{18} \mathrm{H}_{19} \mathrm{~N}_{2} \mathrm{O}$ 279.1497; Found: 279.1414.

3-(4-Chlorobenzyl)-3-hydro-2-methoxy-5-methyl-isoindolin-1-one, 4db. Colorless solid (20 mg, $0.066 \mathrm{mmol}, 22 \%) .{ }^{1} \mathrm{H} \mathrm{NMR}\left(\mathrm{CDCl}_{3}, 400 \mathrm{MHz}\right): \delta 7.60(\mathrm{~d}, 1 \mathrm{H}, J=7.7 \mathrm{~Hz}), 7.23-7.14(\mathrm{~m}, 3 \mathrm{H}), 7.02-$ $6.97(\mathrm{~m}, 2 \mathrm{H}, \mathrm{Ar}-\mathrm{H}), 6.92-6.89(\mathrm{~m}, 1 \mathrm{H}), 4.92(\mathrm{dd}, 1 \mathrm{H}, J=6.5,4.3 \mathrm{~Hz}), 3.95(\mathrm{~s}, 3 \mathrm{H}), 3.29(\mathrm{dd}, 1 \mathrm{H}, J=$ 13.9, $4.3 \mathrm{~Hz}), 3.12(\mathrm{dd}, 1 \mathrm{H}, J=14.0,6.5 \mathrm{~Hz}), 2.39$ (s, 3H). ${ }^{13} \mathrm{C} \mathrm{NMR}\left(\mathrm{CDCl}_{3}, 101 \mathrm{MHz}\right): \delta 164.9,142.7$, $141.5,133.8,132.9,131.2,129.7,128.5,127.4,123.8,123.3,63.8,59.8,36.9,22.2$. HRMS (ESI-TOF) m/z: $[\mathrm{M}+\mathrm{H}]^{+}$Calcd for $\mathrm{C}_{17} \mathrm{H}_{17} \mathrm{NO}_{2} \mathrm{Cl}$ 302.0947; Found: 302.0933; $\left[\mathrm{M}+\mathrm{H}+\mathrm{CH}_{3} \mathrm{CN}\right]^{+}$Calcd for $\mathrm{C}_{19} \mathrm{H}_{20} \mathrm{~N}_{2} \mathrm{O}_{2} \mathrm{Cl}$ 343.1213; Found: 343.1199.

3-(4-Fluorobenzyl)-3-hydro-2-methoxy-5-methyl-isoindolin-1-one, 4dc. Colorless solid (21 mg, 0.073 mmol, 24\%). mp 95-96 ${ }^{\circ} \mathrm{C} .{ }^{1} \mathrm{H}$ NMR $\left(\mathrm{CDCl}_{3}, 400 \mathrm{MHz}\right): \delta 7.57(\mathrm{~d}, 1 \mathrm{H}, J=7.8 \mathrm{~Hz}), 7.18(\mathrm{ddt}, 1 \mathrm{H}, J=$ 7.8, 1.5, 0.7 Hz), 7.04-6.96 (m, 2H), 6.91-6.82 (m, 3H), $4.91(\mathrm{dd}, 1 \mathrm{H}, J=6.6,4.3 \mathrm{~Hz}), 3.94(\mathrm{~s}, 3 \mathrm{H}), 3.29$ $(\mathrm{dd}, 1 \mathrm{H}, J=14.0,4.3 \mathrm{~Hz}), 3.10(\mathrm{dd}, 1 \mathrm{H}, J=14.0,6.6 \mathrm{~Hz}), 2.37(\mathrm{~s}, 3 \mathrm{H}) .{ }^{13} \mathrm{C} \mathrm{NMR}\left(\mathrm{CDCl}_{3}, 101 \mathrm{MHz}\right)$ : $\delta 164.7,162.0(\mathrm{~d}, J=245 \mathrm{~Hz}), 142.4,141.5,131.3(\mathrm{~d}, J=8.0 \mathrm{~Hz}), 130.8,129.4,127.3,123.6,123.3$, 
$115.1(\mathrm{~d}, J=21 \mathrm{~Hz}), 63.6,59.8,36.6,22.0$. HRMS (ESI-TOF) m/z: $[\mathrm{M}+\mathrm{H}]^{+}$Calcd for $\mathrm{C}_{17} \mathrm{H}_{17} \mathrm{NO}_{2} \mathrm{~F}$ 286.1243; Found: 286.1237; [M+H+CH $\left.3{ }_{3} \mathrm{CN}\right]^{+}$Calcd for $\mathrm{C}_{19} \mathrm{H}_{20} \mathrm{~N}_{2} \mathrm{O}_{2} \mathrm{~F}$ 327.1509; Found: 327.1559 .

3-(4-Fluorobenzyl)-2,3-dihydro-5-methyl-isoindolin-1-one, 4'dc. Colorless solid (10 mg, 0.039 mmol, 13\%). mp 191-193 ${ }^{\circ} \mathrm{C} .{ }^{1} \mathrm{H}$ NMR $\left(\mathrm{CDCl}_{3}, 400 \mathrm{MHz}\right): \delta 7.69(\mathrm{~d}, 1 \mathrm{H}, J=7.8 \mathrm{~Hz}), 7.31-7.24(\mathrm{~m}, 1 \mathrm{H}), 7.19-$ $7.10(\mathrm{~m}, 3 \mathrm{H}), 7.01-6.93(\mathrm{~m}, 3 \mathrm{H}), 4.73(\mathrm{dd}, 1 \mathrm{H}, J=8.5,5.2 \mathrm{~Hz}), 3.18(\mathrm{dd}, 1 \mathrm{H}, J=13.7,5.2 \mathrm{~Hz}), 2.81$ (dd, $1 \mathrm{H}, J=13.7,8.5 \mathrm{~Hz}), 2.45(\mathrm{~s}, 3 \mathrm{H}) .{ }^{13} \mathrm{C} \mathrm{NMR}\left(\mathrm{CDCl}_{3}, 101 \mathrm{MHz}\right): \delta 170.8,162.1(\mathrm{~d}, J=245 \mathrm{~Hz}), 147.2$, $142.6,132.7(\mathrm{~d}, J=3.2 \mathrm{~Hz}), 130.9(\mathrm{~d}, J=7.9 \mathrm{~Hz}), 129.6,129.5,123.8,123.3,115.7(\mathrm{~d}, J=22 \mathrm{~Hz}), 57.9$, 40.6, 22.1. HRMS (ESI-TOF) $\mathrm{m} / \mathrm{z}$ : $[\mathrm{M}+\mathrm{H}]^{+}$Calcd for $\mathrm{C}_{16} \mathrm{H}_{15} \mathrm{NOF}$ 256.1137; Found: 256.1151; $\left[\mathrm{M}+\mathrm{H}+\mathrm{CH}_{3} \mathrm{CN}\right]^{+}$Calcd for $\mathrm{C}_{18} \mathrm{H}_{18} \mathrm{~N}_{2} \mathrm{OF} 297.1403$; Found: 297.1427.

3-Hydro-2-methoxy-5-methyl-3-(4-methylbenzyl)-isoindolin-1-one, 4dd. Colorless solid (22 mg, $0.078 \mathrm{mmol}, 26 \%) .{ }^{1} \mathrm{H} \mathrm{NMR}\left(\mathrm{CDCl}_{3}, 400 \mathrm{MHz}\right): \delta 7.61(\mathrm{~d}, 1 \mathrm{H}, J=7.8 \mathrm{~Hz}), 7.20-7.17(\mathrm{~m}, 1 \mathrm{H}), 7.05-$ $6.96(\mathrm{~m}, 4 \mathrm{H}), 6.82-6.79(\mathrm{~m}, 1 \mathrm{H}), 4.89(\mathrm{dd}, 1 \mathrm{H}, J=7.4,4.4 \mathrm{~Hz}), 3.95(\mathrm{~s}, 3 \mathrm{H}), 3.36(\mathrm{dd}, 1 \mathrm{H}, J=13.8,4.4$ $\mathrm{Hz}), 2.98(\mathrm{dd}, 1 \mathrm{H}, J=13.8,7.4 \mathrm{~Hz}), 2.36(\mathrm{~s}, 3 \mathrm{H}), 2.28(\mathrm{~s}, 3 \mathrm{H}) .{ }^{13} \mathrm{C} \mathrm{NMR}\left(\mathrm{CDCl}_{3}, 101 \mathrm{MHz}\right): \delta 165.0$, $142.4,142.1,136.5,132.4,129.7,129.4,129.1,127.4,123.6,63.8,60.4,37.4,22.1,21.2$. HRMS (ESITOF) m/z: $[\mathrm{M}+\mathrm{H}]^{+}$Calcd for $\mathrm{C}_{18} \mathrm{H}_{20} \mathrm{NO}_{2}$ 282.1494; Found: 282.1485; $\left[\mathrm{M}+\mathrm{Na}+\mathrm{CH}_{3} \mathrm{CN}\right]^{+} \mathrm{Calcd}$ for $\mathrm{C}_{20} \mathrm{H}_{22} \mathrm{~N}_{2} \mathrm{O}_{2} \mathrm{Na} 345.1579$; Found: 345.1548.

2,3-Dihydro-5-methyl-3-(4-methylbenzyl)isoindolin-1-one, 4'dd. Colorless solid (8 mg, $0.031 \mathrm{mmol}$, 10\%). mp 165-166 ${ }^{\circ} \mathrm{C} .{ }^{1} \mathrm{H}$ NMR $\left(\mathrm{CDCl}_{3}, 400 \mathrm{MHz}\right): \delta 7.72(\mathrm{~d}, 1 \mathrm{H}, J=7.8 \mathrm{~Hz}), 7.28(\mathrm{ddd}, 1 \mathrm{H}, J=8.4$, 1.4, 0.6 Hz), 7.21-7.18 (m, 1H), 7.18- $7.10(\mathrm{~m}, 4 \mathrm{H}), 6.15$ (br s, 1H), 4.70 (dd, 1H, J = 9.8, 4.5 Hz), 3.30$3.15(\mathrm{~m}, 1 \mathrm{H}), 2.64(\mathrm{dd}, 1 \mathrm{H}, J=13.6,9.8 \mathrm{~Hz}), 2.46(\mathrm{~s}, 3 \mathrm{H}), 2.35(\mathrm{~s}, 3 \mathrm{H}) .{ }^{13} \mathrm{C} \mathrm{NMR}\left(\mathrm{CDCl}_{3}, 101 \mathrm{MHz}\right)$ : $\delta 170.4,147.5,142.6,136.9,134.3,129.7,129.5,129.4,129.1,123.8,123.2,58.1,41.3,22.1,21.2$. HRMS (ESI-TOF) m/z: $[\mathrm{M}+\mathrm{H}]^{+}$Calcd for $\mathrm{C}_{17} \mathrm{H}_{18} \mathrm{NO}$ 252.1388; Found: 252.1400; $\left[\mathrm{M}+\mathrm{H}+\mathrm{CH}_{3} \mathrm{CN}^{+}\right.$ Calcd for $\mathrm{C}_{19} \mathrm{H}_{21} \mathrm{~N}_{2} \mathrm{O}$ 293.1654; Found: 293.1678 . 
3-Hydro-2-methoxy-5-methyl-3-(2-naphthylmethyl)-isoindolin-1-one, 4de. Colorless solid (28 mg, $0.088 \mathrm{mmol}, 29 \%) .{ }^{1} \mathrm{H}$ NMR $\left(\mathrm{CDCl}_{3}, 400 \mathrm{MHz}\right): \delta 7.82-7.71(\mathrm{~m}, 3 \mathrm{H}), 7.64-7.57(\mathrm{~m}, 2 \mathrm{H}), 7.48-7.41(\mathrm{~m}$, 2H), 7.28-7.25 (m, 1H), $7.18(\mathrm{ddt}, 1 \mathrm{H}, J=7.8,1.5,0.7 \mathrm{~Hz}), 6.81-6.78(\mathrm{~m}, 1 \mathrm{H}), 5.01(\mathrm{dd}, 1 \mathrm{H}, J=7.5,4.6$ $\mathrm{Hz}), 3.97(\mathrm{~s}, 3 \mathrm{H}), 3.57(\mathrm{dd}, 1 \mathrm{H}, J=13.8,4.6 \mathrm{~Hz}), 3.16(\mathrm{dd}, 1 \mathrm{H}, J=13.8,7.5 \mathrm{~Hz}), 2.31(\mathrm{~s}, 3 \mathrm{H}) .{ }^{13} \mathrm{C} \mathrm{NMR}$ $\left(\mathrm{CDCl}_{3}, 101 \mathrm{MHz}\right): \delta 165.0,142.5,142.1,133.4,133.3,132.5,129.5,128.6,128.1,127.8,127.7,127.3$, 126.2, 125.8, 123.7, 123.6, 63.9, 60.3, 38.1, 22.1. HRMS (ESI-TOF) m/z: $[\mathrm{M}+\mathrm{H}]^{+}$Calcd for $\mathrm{C}_{21} \mathrm{H}_{20} \mathrm{NO}_{2}$ 318.1494; Found: 318.1482.

2,3-Dihydro-5-methyl-3-(2-naphthylmethyl)-isoindolin-1-one, 4'de. Colorless solid (14 mg, 0.048 mmol, 16\%). mp 179-180 ${ }^{\circ} \mathrm{C} .{ }^{1} \mathrm{H}$ NMR $\left(\mathrm{CDCl}_{3}, 400 \mathrm{MHz}\right): \delta 7.83(\mathrm{dd}, 2 \mathrm{H}, J=9.0,2.8 \mathrm{~Hz}), 7.80-7.75$ (m, 1H), $7.72(\mathrm{~d}, 1 \mathrm{H}, J=7.8 \mathrm{~Hz}), 7.69-7.66(\mathrm{~m}, 1 \mathrm{H}), 7.53-7.44(\mathrm{~m}, 2 \mathrm{H}), 7.37(\mathrm{dd}, 1 \mathrm{H}, J=8.4,1.8 \mathrm{~Hz})$, $7.29(\mathrm{dd}, 1 \mathrm{H}, J=7.8,1.3 \mathrm{~Hz}), 7.21(\mathrm{dd}, 1 \mathrm{H}, J=1.5,0.8 \mathrm{~Hz}), 6.51$ (br s, $1 \mathrm{H}), 4.82(\mathrm{dd}, 1 \mathrm{H}, J=9.5,4.7$ $\mathrm{Hz}), 3.41(\mathrm{dd}, 1 \mathrm{H}, J=13.6,4.7 \mathrm{~Hz}), 2.87(\mathrm{dd}, 1 \mathrm{H}, J=13.6,9.5 \mathrm{~Hz}), 2.46(\mathrm{~s}, 3 \mathrm{H}) .{ }^{13} \mathrm{C} \mathrm{NMR}\left(\mathrm{CDCl}_{3}, 101\right.$ MHz): $\delta 170.5,147.5,142.7,134.8,133.7,132.6,129.6,129.4,128.7,128.0,127.8,127.7,127.2,126.5$, 126.0, 123.9, 123.2, 58.0, 41.9, 22.1. HRMS (ESI-TOF) m/z: $[\mathrm{M}+\mathrm{H}]^{+}$Calcd for $\mathrm{C}_{20} \mathrm{H}_{18} \mathrm{NO} 288.1388$; Found: 288.1378; [M+H+CH $3 \mathrm{CN}^{+}$Calcd for $\mathrm{C}_{22} \mathrm{H}_{21} \mathrm{~N}_{2} \mathrm{O} 329.1654$; Found: 329.1651; [M+Na+CH $3 \mathrm{CN}^{+}$ Calcd forC $\mathrm{C}_{22} \mathrm{H}_{20} \mathrm{~N}_{2} \mathrm{ONa} 351.1473$; Found: 351.1459 .

3-Benzyl-3-hydro-2-methoxy-6-methyl-isoindolin-1-one, 4ga. Colorless solid (27 mg, $0.102 \mathrm{mmol}$, 34\%). mp 105-106 ${ }^{\circ} \mathrm{C} .{ }^{1} \mathrm{H}$ NMR $\left(\mathrm{CDCl}_{3}, 400 \mathrm{MHz}\right): \delta 7.54-7.52(\mathrm{~m}, 1 \mathrm{H}), 7.25-7.17(\mathrm{~m}, 4 \mathrm{H}), 7.12-7.08$ $(\mathrm{m}, 2 \mathrm{H}), 6.83(\mathrm{~d}, 1 \mathrm{H}, J=7.7 \mathrm{~Hz}), 4.92(\mathrm{dd}, 1 \mathrm{H}, J=7.6,4.4 \mathrm{~Hz}), 3.96(\mathrm{~s}, 3 \mathrm{H}), 3.42(\mathrm{dd}, 1 \mathrm{H}, J=13.7,4.4$ $\mathrm{Hz}), 2.96(\mathrm{dd}, 1 \mathrm{H}, J=13.7,7.6 \mathrm{~Hz}), 2.35(\mathrm{~s}, 3 \mathrm{H}) .{ }^{13} \mathrm{C} \mathrm{NMR}\left(\mathrm{CDCl}_{3}, 101 \mathrm{MHz}\right): \delta 164.7,138.8,138.5$, 135.6, 132.6, 132.6, 129.8, 128.4, 126.9, 123.9, 122.8, 63.8, 60.2, 37.8, 21.4. HRMS (ESI-TOF) m/z: $[\mathrm{M}+\mathrm{H}]^{+}$Calcd for $\mathrm{C}_{17} \mathrm{H}_{18} \mathrm{NO}_{2}$ 268.1337; Found: 268.1342; $\left[\mathrm{M}+\mathrm{Na}+\mathrm{CH}_{3} \mathrm{CN}\right]^{+}$Calcd forC ${ }_{19} \mathrm{H}_{20} \mathrm{~N}_{2} \mathrm{O}_{2}$ Na333.1422; Found: 331.1426. 
3-Benzyl-2,3-dihydro-6-methyl-isoindolin-1-one, 4'ga. Colorless solid (17 mg, 0.069 mmol, 23\%). mp 131-133 ${ }^{\circ} \mathrm{C} .{ }^{1} \mathrm{H}$ NMR $\left(\mathrm{CDCl}_{3}, 400 \mathrm{MHz}\right): \delta 7.65-7.62(\mathrm{~m}, 1 \mathrm{H}), 7.40-7.26(\mathrm{~m}, 4 \mathrm{H}), 7.25-7.17(\mathrm{~m}, 3 \mathrm{H})$, $6.58($ br s, $1 \mathrm{H}), 4.75(\mathrm{dd}, 1 \mathrm{H}, J=9.1,5.2 \mathrm{~Hz}), 3.19(\mathrm{dd}, 1 \mathrm{H}, J=13.6,5.2 \mathrm{~Hz}), 2.78(\mathrm{dd}, 1 \mathrm{H}, J=13.6,9.1$ $\mathrm{Hz}), 2.44$ (s, 3H). ${ }^{13} \mathrm{C} \mathrm{NMR}\left(\mathrm{CDCl}_{3}, 101 \mathrm{MHz}\right): \delta 170.6,144.3,138.5,137.2,133.7,132.9,132.1,129.3$, 129.0, 127.2, 124.2, 122.5, 57.9, 41.7, 21.5. HRMS (ESI-TOF) $\mathrm{m} / \mathrm{z}:[\mathrm{M}+\mathrm{H}]^{+}$Calcd for $\mathrm{C}_{16} \mathrm{H}_{16} \mathrm{NO}$ 238.1231; Found: 238.1229; $\left[\mathrm{M}+\mathrm{H}+\mathrm{CH}_{3} \mathrm{CN}\right]^{+}$Calcd for $\mathrm{C}_{18} \mathrm{H}_{19} \mathrm{~N}_{2} \mathrm{O} 279.1497$; Found: 279.1508; $\left[\mathrm{M}+\mathrm{Na}+\mathrm{CH}_{3} \mathrm{CN}\right]^{+}$Calcd forC ${ }_{18} \mathrm{H}_{18} \mathrm{~N}_{2} \mathrm{ONa} 301.1317$; Found: 301.1316.

3-(4-Chlorobenzyl)-3-hydro-2-methoxy-6-methyl-isoindolin-1-one, 4gb. Colorless solid (24 mg, $0.079 \mathrm{mmol}, 26 \%) .{ }^{1} \mathrm{H}$ NMR $\left(\mathrm{CDCl}_{3}, 400 \mathrm{MHz}\right): \delta 7.55-7.52(\mathrm{~m}, 1 \mathrm{H}), 7.29-7.26(\mathrm{~m}, 1 \mathrm{H}), 7.19-7.14(\mathrm{~m}$, 2H), 7.04-6.92 (m, 3H), $4.93(\mathrm{dd}, 1 \mathrm{H}, J=6.9,4.2 \mathrm{~Hz}), 3.97(\mathrm{~s}, 3 \mathrm{H}), 3.33(\mathrm{dd}, 1 \mathrm{H}, J=13.9,4.2 \mathrm{~Hz}), 3.06$ $(\mathrm{dd}, 1 \mathrm{H}, J=13.9,6.9 \mathrm{~Hz}), 2.38(\mathrm{~s}, 3 \mathrm{H}) .{ }^{13} \mathrm{C} \mathrm{NMR}\left(\mathrm{CDCl}_{3}, 101 \mathrm{MHz}\right): \delta 164.58,138.7,138.2,133.6$, 132.8, 132.7, 131.1, 130.0, 128.4, 124.1, 122.5, 63.7, 59.6, 36.8, 21.4. HRMS (ESI-TOF) m/z: $[\mathrm{M}+\mathrm{H}]^{+}$ Calcd for $\mathrm{C}_{17} \mathrm{H}_{17} \mathrm{NO}_{2} \mathrm{Cl}$ 302.0947; Found: 302.0939; [M+H+C $\left.\mathrm{CH}_{3} \mathrm{CN}\right]^{+}$Calcd for $\mathrm{C}_{19} \mathrm{H}_{20} \mathrm{~N}_{2} \mathrm{O}_{2} \mathrm{Cl}$ 343.1213; Found: 343.1205; [M+Na+CH $\left.3{ }_{3} \mathrm{CN}\right]^{+}$Calcd forC ${ }_{19} \mathrm{H}_{19} \mathrm{~N}_{2} \mathrm{O}_{2} \mathrm{ClNa} 365.1033$; Found: 365.1009 .

3-Hydro-2-methoxy-5-methyl-3-(4-methylbenzyl)-isoindolin-1-one, 4gd. Colorless solid (40 mg, $0.142 \mathrm{mmol}, 47 \%) .{ }^{1} \mathrm{H}$ NMR $\left(\mathrm{CDCl}_{3}, 400 \mathrm{MHz}\right): \delta 7.55-7.52(\mathrm{~m}, 1 \mathrm{H}), 7.23-7.21(\mathrm{~m}, 1 \mathrm{H}), 7.05-6.96(\mathrm{~m}$, 4H), $6.84(\mathrm{~d}, 1 \mathrm{H}, J=7.8 \mathrm{~Hz}), 4.89(\mathrm{dd}, 1 \mathrm{H}, J=7.8,4.3 \mathrm{~Hz}), 3.97(\mathrm{~s}, 3 \mathrm{H}), 3.40(\mathrm{dd}, 1 \mathrm{H}, J=13.7,4.3 \mathrm{~Hz})$, $2.90(\mathrm{dd}, 1 \mathrm{H}, J=13.7,7.8 \mathrm{~Hz}), 2.36(\mathrm{~s}, 3 \mathrm{H}), 2.28(\mathrm{~s}, 3 \mathrm{H}) .{ }^{13} \mathrm{C} \mathrm{NMR}\left(\mathrm{CDCl}_{3}, 101 \mathrm{MHz}\right): \delta 164.8,138.9$, $138.5,136.5,132.6,132.4,130.0,129.6,129.1,124.0,122.9,63.8,60.3,37.4,21.4,21.2$. HRMS (ESITOF) m/z: $[\mathrm{M}+\mathrm{H}]^{+}$Calcd for $\mathrm{C}_{18} \mathrm{H}_{20} \mathrm{NO}_{2} 282.1494$; Found: 282.1497.

2,3-Dihydro-6-methyl-3-(4-methylbenzyl)-isoindolin-1-one, 4'gd. Colorless solid (7 mg, 0.027 mmol, 9\%). ${ }^{1} \mathrm{H} \mathrm{NMR}\left(\mathrm{CDCl}_{3}, 400 \mathrm{MHz}\right): \delta 7.65-7.63(\mathrm{~m}, 1 \mathrm{H}), 7.38-7.33(\mathrm{~m}, 1 \mathrm{H}), 7.22(\mathrm{~d}, 1 \mathrm{H}, J=7.8 \mathrm{~Hz})$, 7.17-7.10 (m, 4H), 6.36 (br s, 1H), $4.72(\mathrm{dd}, 1 \mathrm{H}, J=9.3,5.0 \mathrm{~Hz}), 3.20-3.14(\mathrm{~m}, 1 \mathrm{H}), 2.70(\mathrm{dd}, 1 \mathrm{H}, J$ 
$=13.6,9.3 \mathrm{~Hz}), 2.44(\mathrm{~s}, 3 \mathrm{H}), 2.35(\mathrm{~s}, 3 \mathrm{H}) .{ }^{13} \mathrm{C} \mathrm{NMR}\left(\mathrm{CDCl}_{3}, 101 \mathrm{MHz}\right): \delta 170.5,144.4,138.5,136.9$,

134.2, 132.9, 132.1, 129.7, 129.2, 124.2, 122.5, 58.1, 41.3, 21.5, 21.2. HRMS (ESI-TOF) m/z: $[\mathrm{M}+\mathrm{H}]^{+}$ Calcd for $\mathrm{C}_{17} \mathrm{H}_{18} \mathrm{NO}$ 252.1388; Found: 252.1393; $\left[\mathrm{M}+\mathrm{H}+\mathrm{CH}_{3} \mathrm{CN}\right]^{+}$Calcd for $\mathrm{C}_{19} \mathrm{H}_{21} \mathrm{~N}_{2} \mathrm{O}$ 293.1654; Found: 293.1649; [M+Na+CH $\left.3{ }_{3} \mathrm{CN}\right]^{+}$Calcd forC ${ }_{19} \mathrm{H}_{20} \mathrm{~N}_{2} \mathrm{ONa}$ 315.1473; Found: 315.1470 .

3-Hydro-2-methoxy-6-methyl-3-(2-naphthylmethyl)-isoindolin-1-one, 4ge. Colorless solid (34 mg, $0.107 \mathrm{mmol}, 36 \%) .{ }^{1} \mathrm{H}$ NMR $\left(\mathrm{CDCl}_{3}, 400 \mathrm{MHz}\right): \delta 7.82-7.72(\mathrm{~m}, 3 \mathrm{H}), 7.60-7.53(\mathrm{~m}, 2 \mathrm{H}), 7.47-7.41(\mathrm{~m}$, 2H), 7.31-7.24 (m, 2H), 7.18 (ddd, 1H, $J=7.9,1.7,0.8 \mathrm{~Hz}), 6.81(\mathrm{~d}, 1 \mathrm{H}, J=7.7 \mathrm{~Hz}), 5.02(\mathrm{dd}, 1 \mathrm{H}, J=$ 7.9, $4.5 \mathrm{~Hz}), 3.99$ (s, 3H), $3.62(\mathrm{dd}, 1 \mathrm{H}, J=13.8,4.5 \mathrm{~Hz}), 3.09$ (dd, 1H, $J=13.8,7.9 \mathrm{~Hz}), 2.34(\mathrm{~s}, 3 \mathrm{H})$. ${ }^{13} \mathrm{C}$ NMR $\left(\mathrm{CDCl}_{3}, 101 \mathrm{MHz}\right): \delta 164.8,138.9,138.6,133.4,133.3,132.7,132.5,129.9,128.6,128.1$, $127.8,127.7,126.2,125.8,124.1,122.9,63.9,60.2,38.1,21.4$. HRMS (ESI-TOF) m/z: $[\mathrm{M}+\mathrm{H}]^{+} \mathrm{Calcd}$ for $\mathrm{C}_{21} \mathrm{H}_{20} \mathrm{NO}_{2}$ 318.1494; Found: 318.1495 .

2,3-Dihydro-6-methyl-3-(2-naphthylmethyl)-isoindolin-1-one, 4'ge. Colorless solid (6 mg, 0.020 mmol, 7\%). mp 159-160 ${ }^{\circ} \mathrm{C} .{ }^{1} \mathrm{H} \mathrm{NMR}\left(\mathrm{CDCl}_{3}, 400 \mathrm{MHz}\right): \delta 7.88-7.76(\mathrm{~m}, 3 \mathrm{H}), 7.71-7.63(\mathrm{~m}, 2 \mathrm{H}), 7.53-$ $7.45(\mathrm{~m}, 2 \mathrm{H}), 7.42-7.33(\mathrm{~m}, 2 \mathrm{H}), 7.25(\mathrm{~d}, 1 \mathrm{H}, J=7.7 \mathrm{~Hz}), 6.42(\mathrm{br} \mathrm{s}, 1 \mathrm{H}), 4.84$ (dd, $1 \mathrm{H}, J=9.4,5.0 \mathrm{~Hz})$, $3.37(\mathrm{dd}, 1 \mathrm{H}, J=13.6,5.0 \mathrm{~Hz}), 2.90(\mathrm{dd}, 1 \mathrm{H}, J=13.6,9.4 \mathrm{~Hz}), 2.45(\mathrm{~s}, 3 \mathrm{H}) .{ }^{13} \mathrm{C} \mathrm{NMR}\left(\mathrm{CDCl}_{3}, 101 \mathrm{MHz}\right)$ : $\delta 170.5,144.3,138.6,134.8,133.7,133.0,132.6,132.1,128.8,128.1,127.9,127.7,127.3,126.5,126.0$ 124.3, 122.5, 58.0, 42.0, 21.5. HRMS (ESI-TOF) m/z: $[\mathrm{M}+\mathrm{H}]^{+}$Calcd for $\mathrm{C}_{20} \mathrm{H}_{18} \mathrm{NO}$ 288.1388; Found: 288.1400; $\left[\mathrm{M}+\mathrm{H}+\mathrm{CH}_{3} \mathrm{CN}\right]^{+}$Calcd for $\mathrm{C}_{22} \mathrm{H}_{21} \mathrm{~N}_{2} \mathrm{O}$ 329.1653; Found: 329.1673; [M+Na+CH$\left.{ }_{3} \mathrm{CN}\right]^{+} \mathrm{Calcd}$ for $\mathrm{C}_{22} \mathrm{H}_{20} \mathrm{~N}_{2} \mathrm{ONa} 351.1473$ Found: 351.1480 .

3-(4-Trifluoromethyl-benzyl)-3-hydro-2-methoxy-6-methyl-isoindolin-1-one, 4gf. Colorless solid (23 $\mathrm{mg}, 0.069 \mathrm{mmol}, 23 \%) .{ }^{1} \mathrm{H}$ NMR $\left(\mathrm{CDCl}_{3}, 400 \mathrm{MHz}\right): \delta$ 7.54-7.51 (m, 1H), 7.47-7.43 (m, 2H), 7.28 (ddd, $1 \mathrm{H}, J=7.7,1.7,0.8 \mathrm{~Hz}), 7.21-7.16(\mathrm{~m}, 2 \mathrm{H}), 6.96(\mathrm{~d}, 1 \mathrm{H}, J=7.8 \mathrm{~Hz}), 4.98(\mathrm{dd}, 1 \mathrm{H}, J=6.8,4.3 \mathrm{~Hz}), 3.97$ (s, 3H), 3.44-3.36 (m, 1H), 3.17 (dd, $1 \mathrm{H}, J=13.8,6.8 \mathrm{~Hz}), 2.37$ (s, 3H). $\left.{ }^{13} \mathrm{C} \mathrm{NMR} \mathrm{(CDCl}, 101 \mathrm{MHz}\right): \delta$ 
$164.7,139.5,139.0,138.2,132.9,131.0,130.2,130.1,125.3(\mathrm{q}, J=3.6 \mathrm{~Hz}), 124.3,124.2,122.6,63.8$, 59.6, 37.4, 21.5. HRMS (ESI-TOF) m/z: $[\mathrm{M}+\mathrm{H}]^{+}$Calcd for $\mathrm{C}_{18} \mathrm{H}_{17} \mathrm{NO}_{2} \mathrm{~F}_{3}$ 336.1211; Found: 336.1205; $\left[\mathrm{M}+\mathrm{H}+\mathrm{CH}_{3} \mathrm{CN}\right]^{+}$Calcd for $\mathrm{C}_{20} \mathrm{H}_{20} \mathrm{~N}_{2} \mathrm{O}_{2} \mathrm{~F}_{3} 377.1477$; Found: 377.1468 .

3-(4-Trifluoromethyl-benzyl)-2,3-dihydro-6-methyl-isoindolin-1-one, 4'gf. Colorless solid (27 mg, $0.087 \mathrm{mmol}, 30 \%) .{ }^{1} \mathrm{H} \mathrm{NMR}\left(\mathrm{CDCl}_{3}, 400 \mathrm{MHz}\right): \delta 7.63-7.60(\mathrm{~m}, 1 \mathrm{H}), 7.56(\mathrm{~d}, 2 \mathrm{H}, J=7.9 \mathrm{~Hz}), 7.37$ (ddd, 1H, $J=7.8,1.7,0.8 \mathrm{~Hz}), 7.32(\mathrm{~d}, 2 \mathrm{H}, J=8.0 \mathrm{~Hz}), 7.20(\mathrm{~d}, 1 \mathrm{H}, J=7.7 \mathrm{~Hz}), 6.89$ (s, 1H), 4.79 (dd, $1 \mathrm{H}, J=8.3,5.3 \mathrm{~Hz}), 3.25(\mathrm{dd}, 1 \mathrm{H}, J=13.6,5.3 \mathrm{~Hz}), 2.91(\mathrm{dd}, 1 \mathrm{H}, J=13.6,8.3 \mathrm{~Hz}), 2.44(\mathrm{~s}, 3 \mathrm{H}) .{ }^{13} \mathrm{C}$ NMR ( $\left.\mathrm{CDCl}_{3}, 101 \mathrm{MHz}\right): \delta 170.8,143.8,141.1,138.9,133.1,132.1,129.8,125.8(\mathrm{q}, J=3.7 \mathrm{~Hz}), 125.6$, 124.4, 122.4, 57.5, 41.3, 21.5. HRMS (ESI-TOF) m/z: $[\mathrm{M}+\mathrm{H}]^{+}$Calcd for $\mathrm{C}_{17} \mathrm{H}_{15} \mathrm{NOF}_{3} 306.1105$; Found: 306.1115; $\left[\mathrm{M}+\mathrm{H}+\mathrm{CH}_{3} \mathrm{CN}\right]^{+}$Calcd for $\mathrm{C}_{19} \mathrm{H}_{18} \mathrm{~N}_{2} \mathrm{OF}_{3}$ 347.1371; Found: 347.1367.

3-Benzyl-3-hydro-2,6-dimethoxy-isoindolin-1-one, 4ha. Colorless solid (21 mg, $0.072 \mathrm{mmol}, 23 \%$ ). mp 100-101 ${ }^{\circ} \mathrm{C} .{ }^{1} \mathrm{H}$ NMR $\left(\mathrm{CDCl}_{3}, 400 \mathrm{MHz}\right): \delta 7.25-7.19(\mathrm{~m}, 3 \mathrm{H}), 7.12-7.08(\mathrm{~m}, 2 \mathrm{H}), 6.97(\mathrm{dd}, 1 \mathrm{H}, J$ $=8.4,2.5 \mathrm{~Hz}), 6.83(\mathrm{dt}, 1 \mathrm{H}, J=8.4,0.6 \mathrm{~Hz}), 4.91(\mathrm{ddd}, 1 \mathrm{H}, J=7.7,4.3,0.7 \mathrm{~Hz}), 3.98(\mathrm{~s}, 3 \mathrm{H}), 3.79(\mathrm{~s}$, 3H), $3.43(\mathrm{dd}, 1 \mathrm{H}, J=13.7,4.3 \mathrm{~Hz}), 2.95(\mathrm{dd}, 1 \mathrm{H}, J=13.7,7.7 \mathrm{~Hz}) .{ }^{13} \mathrm{C} \mathrm{NMR}\left(\mathrm{CDCl}_{3}, 101 \mathrm{MHz}\right)$ : $\delta 164.5,160.0,135.4,133.7,131.2,129.7,128.4,126.9,124.1,119.8,106.6,63.8,60.0,55.6,37.8$. HRMS (ESI-TOF) m/z: $[\mathrm{M}+\mathrm{H}]^{+}$Calcd for $\mathrm{C}_{17} \mathrm{H}_{18} \mathrm{NO}_{3} 284.1286$; Found: 284.1276.

3-Benzyl-2,3-dihydro-6-methoxy-isoindolin-1-one, 4'ha. Colorless solid (23 mg, $0.091 \mathrm{mmol,} 29 \%$ ). ${ }^{1} \mathrm{H}$ NMR $\left(\mathrm{CDCl}_{3}, 400 \mathrm{MHz}\right): \delta 7.39-7.28(\mathrm{~m}, 4 \mathrm{H}), 7.26-7.21(\mathrm{~m}, 3 \mathrm{H}), 7.11(\mathrm{dd}, 1 \mathrm{H}, J=8.3,2.5 \mathrm{~Hz})$, 6.15 (br s, 1H), 4.71-4.75 (m, 1H), $3.86(\mathrm{~s}, 3 \mathrm{H}), 3.22(\mathrm{dd}, 1 \mathrm{H}, J=13.4,5.0 \mathrm{~Hz}), 2.72(\mathrm{dd}, 1 \mathrm{H}, J=13.5$, $9.4 \mathrm{~Hz}) .{ }^{13} \mathrm{C} \mathrm{NMR}\left(\mathrm{CDCl}_{3}, 101 \mathrm{MHz}\right): \delta 170.1,160.2,139.1,137.1,133.2,129.1,128.9,127.2,123.5$, 120.3, 106.5, 57.6, 55.7, 41.7. HRMS (ESI-TOF) m/z: $[\mathrm{M}+\mathrm{H}]^{+}$Calcd for $\mathrm{C}_{16} \mathrm{H}_{16} \mathrm{NO}_{2} 254.1181$; Found: 254.1179; [M+H+CH $\left.3{ }_{3} \mathrm{CN}\right]^{+}$Calcd for $\mathrm{C}_{18} \mathrm{H}_{19} \mathrm{~N}_{2} \mathrm{O}_{2}$ 295.1446; Found: 295.1449; [M+Na+CH $3 \mathrm{CN}^{+} \mathrm{Calcd}$ for $\mathrm{C}_{18} \mathrm{H}_{18} \mathrm{~N}_{2} \mathrm{O}_{2} \mathrm{Na}$ 317.1266; Found: 317.1256. 
3-Hydro-2,5-dimethoxy-3-(4-methylbenzyl)-isoindolin-1-one, 4hd. Colorless solid (33 mg, 0.111 mmol, $37 \%$ \%). ${ }^{1} \mathrm{H} \mathrm{NMR}\left(\mathrm{CDCl}_{3}, 400 \mathrm{MHz}\right): \delta 7.23(\mathrm{~d}, 1 \mathrm{H}, J=2.4 \mathrm{~Hz}), 7.05-6.95(\mathrm{~m}, 5 \mathrm{H}), 6.85-6.82(\mathrm{~m}$, 1H), 4.88 (ddd, 1H, J=7.8, 4.2, $0.7 \mathrm{~Hz}), 3.98$ (s, 3H), 3.79 (s, 3H), 3.39 (dd, 1H, J =13.7, 4.2 Hz), 2.90 $(\mathrm{dd}, 1 \mathrm{H}, J=13.7,7.8 \mathrm{~Hz}), 2.28(\mathrm{~s}, 3 \mathrm{H}) .{ }^{13} \mathrm{C} \mathrm{NMR}\left(\mathrm{CDCl}_{3}, 101 \mathrm{MHz}\right): \delta 164.6,160.1,136.5,133.9,132.3$, 131.3, 129.7, 129.2, 124.2, 119.8, 106.6, 63.9, 60.1, 55.7, 37.5, 21.2. HRMS (ESI-TOF) m/z: [M+H] ${ }^{+}$ Calcd for $\mathrm{C}_{18} \mathrm{H}_{20} \mathrm{NO}_{3} 298.1443$; Found: 298.1452.

2,3-Dihydro-6-methoxy-3-(4-Methylbenzyl)-isoindolin-1-one, 4'hd. Colorless solid (8 mg, 0.029 mmol, 10\%). mp 147-148 ${ }^{\circ} \mathrm{C} .{ }^{1} \mathrm{H}$ NMR $\left(\mathrm{CDCl}_{3}, 400 \mathrm{MHz}\right): \delta 7.31(\mathrm{~d}, 1 \mathrm{H}, J=2.4 \mathrm{~Hz}), 7.21(\mathrm{~d}, 1 \mathrm{H}, J=$ 8.3Hz), $7.17-7.08(\mathrm{~m}, 5 \mathrm{H}), 6.37($ br s, $1 \mathrm{H}), 4.74-4.65(\mathrm{~m}, 1 \mathrm{H}), 3.86(\mathrm{~s}, 3 \mathrm{H}), 3.20-3.11(\mathrm{~m}, 1 \mathrm{H}), 2.71(\mathrm{dd}$, $1 \mathrm{H}, J=13.6,9.2 \mathrm{~Hz}), 2.35(\mathrm{~s}, 3 \mathrm{H}) .{ }^{13} \mathrm{C} \mathrm{NMR}\left(\mathrm{CDCl}_{3}, 101 \mathrm{MHz}\right): \delta 170.3,160.3,139.4,136.9,134.1$, 133.3, 129.7, 129.2, 123.7, 120.3, 106.6, 77.5, 77.2, 76.8, 57.9, 55.8, 41.3, 21.2. HRMS (ESI-TOF) m/z: $[\mathrm{M}+\mathrm{H}]^{+}$Calcd for $\mathrm{C}_{17} \mathrm{H}_{18} \mathrm{NO}_{2}$ 268.1337; Found: 268.1344; $\left[\mathrm{M}+\mathrm{H}+\mathrm{CH}_{3} \mathrm{CN}\right]^{+}$Calcd for $\mathrm{C}_{19} \mathrm{H}_{21} \mathrm{~N}_{2} \mathrm{O}_{2}$ 309.1603; Found: 309.1613; [M+Na+CH $\left.3{ }_{3} \mathrm{CN}\right]^{+}$Calcd for $\mathrm{C}_{19} \mathrm{H}_{20} \mathrm{~N}_{2} \mathrm{O}_{2} \mathrm{Na}$ 331.1422 Found: 331.1424.

3-Hydro-2,6-dimethoxy-3-(2-naphthylmethyl)-isoindolin-1-one, 4he. Colorless solid (35 mg, 0.105 mmol, 35\%). ${ }^{1} \mathrm{H}$ NMR $\left(\mathrm{CDCl}_{3}, 400 \mathrm{MHz}\right): \delta 7.83-7.71(\mathrm{~m}, 3 \mathrm{H}), 7.58(\mathrm{~d}, 1 \mathrm{H}, J=1.8 \mathrm{~Hz}), 7.48-7.40(\mathrm{~m}$, 2H), 7.30-7.20 (m, 3H), $6.93(\mathrm{dd}, 1 \mathrm{H}, J=8.4,2.5 \mathrm{~Hz}), 6.81(\mathrm{dt}, 1 \mathrm{H}, J=8.3,0.6 \mathrm{~Hz}), 5.01(\mathrm{ddd}, 1 \mathrm{H}, J=$ 7.9, 4.4, 0.7 Hz), $4.01(\mathrm{~s}, 3 \mathrm{H}), 3.77(\mathrm{~s}, 3 \mathrm{H}), 3.61(\mathrm{dd}, 1 \mathrm{H}, J=13.7,4.4 \mathrm{~Hz}), 3.09$ (dd, $1 \mathrm{H}, J=13.7,7.9$ Hz). ${ }^{13} \mathrm{C} \mathrm{NMR}\left(\mathrm{CDCl}_{3}, 101 \mathrm{MHz}\right): \delta 164.7,160.1,133.8,133.4,133.2,132.5,131.3,128.6,128.1,127.8$, 127.7, 126.2, 125.8, 124.2, 119.9, 106.7, 63.9, 60.1, 55.7, 38.2. HRMS (ESI-TOF) m/z: $[\mathrm{M}+\mathrm{H}]^{+}$Calcd for $\mathrm{C}_{21} \mathrm{H}_{20} \mathrm{NO}_{3}$ 334.1443; Found: 334.1428; [M+H+CH $\left.\mathrm{CH}_{3} \mathrm{CN}\right]^{+}$Calcd for $\mathrm{C}_{23} \mathrm{H}_{23} \mathrm{~N}_{2} \mathrm{O}_{3} 375.1708$; Found: 375.1662.

2,3-Dihydro-6-methoxy-3-(2-naphthylmethyl)-isoindolin-1-one, 4'he. Colorless solid (8 mg, 0.026 mmol, 9\%). mp 212-215 ${ }^{\circ} \mathrm{C} .{ }^{1} \mathrm{H}$ NMR $\left(\mathrm{CDCl}_{3}, 400 \mathrm{MHz}\right): \delta 7.89-7.76(\mathrm{~m}, 3 \mathrm{H}), 7.68(\mathrm{~d}, 1 \mathrm{H}, J=1.7 \mathrm{~Hz})$, 
7.55-7.45 (m, 2H), $7.38(\mathrm{dd}, 1 \mathrm{H}, J=8.4,1.8 \mathrm{~Hz}), 7.33(\mathrm{~d}, 1 \mathrm{H}, J=2.4 \mathrm{~Hz}), 7.25-7.20(\mathrm{~m}, 1 \mathrm{H}), 7.11(\mathrm{dd}$, $1 \mathrm{H}, J=8.3,2.5 \mathrm{~Hz}), 6.43($ br s, $1 \mathrm{H}), 4.83(\mathrm{ddd}, 1 \mathrm{H}, J=9.2,5.1,0.9 \mathrm{~Hz}), 3.86(\mathrm{~s}, 3 \mathrm{H}), 3.36(\mathrm{dd}, 1 \mathrm{H}, J=$

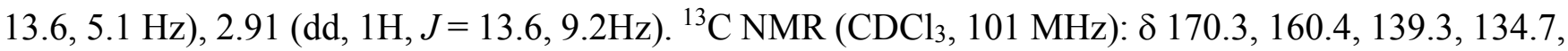
$133.7,133.3,132.6,128.8,128.1,127.9,127.7,127.2,126.6,126.1,123.7,120.4,106.7,57.8,55.8,42.0$. HRMS (ESI-TOF) m/z: [M+H] $]^{+}$Calcd for $\mathrm{C}_{20} \mathrm{H}_{18} \mathrm{NO}_{2}$ 304.1337; Found: 304.1333; $\left[\mathrm{M}+\mathrm{Na}+\mathrm{CH}_{3} \mathrm{CN}^{+}\right.$ Calcd for $\mathrm{C}_{20} \mathrm{H}_{17} \mathrm{NO}_{2} \mathrm{Na} 326.1157$; Found: 326.1151 .

3-(4-Chlorobenzyl)-3-hydro-2,5-dimethoxy-isoindolin-1-one, 4ib. Colorless solid (18 mg, 0.057 mmol, 19\%). ${ }^{1} \mathrm{H} \mathrm{NMR}\left(\mathrm{CDCl}_{3}, 400 \mathrm{MHz}\right): \delta 7.65(\mathrm{~d}, 1 \mathrm{H}, J=8.4 \mathrm{~Hz}), 7.22-7.16(\mathrm{~m}, 2 \mathrm{H}), 7.07-7.01(\mathrm{~m}$, 2H), $6.91(\mathrm{dd}, 1 \mathrm{H}, J=8.4,2.3 \mathrm{~Hz}), 6.59-6.46(\mathrm{~m}, 1 \mathrm{H}), 4.90(\mathrm{dd}, 1 \mathrm{H}, J=7.0,4.4 \mathrm{~Hz}), 3.96(\mathrm{~s}, 3 \mathrm{H}), 3.78$ $(\mathrm{s}, 3 \mathrm{H}), 3.33(\mathrm{dd}, 1 \mathrm{H}, J=13.9,4.4 \mathrm{~Hz}), 3.07(\mathrm{dd}, 1 \mathrm{H}, J=13.9,7.0 \mathrm{~Hz}) .{ }^{13} \mathrm{C} \mathrm{NMR}\left(\mathrm{CDCl}_{3}, 101 \mathrm{MHz}\right)$ : $\delta 165.1,162.9,143.5,133.8,133.0,131.2,128.6,125.6,122.4,114.8,108.3,63.9,60.0,55.7,37.1$. HRMS (ESI-TOF) m/z: [M+H] ${ }^{+}$Calcd for $\mathrm{C}_{17} \mathrm{H}_{17} \mathrm{NO}_{3} \mathrm{Cl}$ 318.0896; Found: 318.0905 .

3-Hydro-2,5-dimethoxy-3-(4-methylbenzyl)-isoindolin-1-one, 4id. Colorless solid (25 mg, 0.084 mmol, 28\%). ${ }^{1} \mathrm{H}$ NMR (CDCl $\left.3,400 \mathrm{MHz}\right): \delta 7.66$ (d, 1H, J=8.4 Hz), 7.08-6.99 (m, 4H), 6.90 (dd, 1H, $J=8.4,2.3 \mathrm{~Hz}), 6.41-6.38(\mathrm{~m}, 1 \mathrm{H}), 4.86(\mathrm{dd}, 1 \mathrm{H}, J=8.0,4.4 \mathrm{~Hz}), 3.96(\mathrm{~s}, 3 \mathrm{H}), 3.72(\mathrm{~s}, 3 \mathrm{H}), 3.42(\mathrm{dd}$, $1 \mathrm{H}, J=13.7,4.4 \mathrm{~Hz}), 2.91(\mathrm{dd}, 1 \mathrm{H}, J=13.7,8.0 \mathrm{~Hz}), 2.29(\mathrm{~s}, 3 \mathrm{H}) .{ }^{13} \mathrm{C} \mathrm{NMR}\left(\mathrm{CDCl}_{3}, 101 \mathrm{MHz}\right): \delta 165.2$, $162.8,144.1,136.6,132.5,129.7,129.2,125.3,122.3,115.0,108.3,64.0,60.6,55.6,37.5,21.2$. HRMS (ESI-TOF) m/z: $[\mathrm{M}+\mathrm{H}]^{+}$Calcd for $\mathrm{C}_{18} \mathrm{H}_{20} \mathrm{NO}_{3}$ 298.1443; Found: 298.1437; [M+Na+CH $3 \mathrm{CN}^{+} \mathrm{Calcd}$ for $\mathrm{C}_{20} \mathrm{H}_{22} \mathrm{~N}_{2} \mathrm{O}_{3} \mathrm{Na}$ 361.1528; Found: 361.1526 .

2,3-Dihydro-5-methoxy-3-(4-methylbenzyl)-isoindolin-1-one, 4'id. Colorless solid (10 mg, 0.037 mmol, 12\%). mp 181-182 ${ }^{\circ} \mathrm{C} .{ }^{1} \mathrm{H}$ NMR $\left(\mathrm{CDCl}_{3}, 400 \mathrm{MHz}\right): \delta 7.74(\mathrm{~d}, 1 \mathrm{H}, J=8.4 \mathrm{~Hz}), 7.19-7.09$ (m, 4H), $6.98(\mathrm{dd}, 1 \mathrm{H}, J=8.4,2.2 \mathrm{~Hz}), 6.81-6.76(\mathrm{~m}, 1 \mathrm{H}), 6.31(\mathrm{br} \mathrm{s}, 1 \mathrm{H}), 4.69(\mathrm{dd}, 1 \mathrm{H}, J=9.2,5.1 \mathrm{~Hz}), 3.84$ $(\mathrm{s}, 3 \mathrm{H}), 3.15(\mathrm{dd}, 1 \mathrm{H}, J=13.5,5.1 \mathrm{~Hz}), 2.74(\mathrm{dd}, 1 \mathrm{H}, J=13.5,9.2 \mathrm{~Hz}), 2.34(\mathrm{~s}, 3 \mathrm{H}) .{ }^{13} \mathrm{C} \mathrm{NMR}\left(\mathrm{CDCl}_{3}\right.$, 
$101 \mathrm{MHz}): \delta 170.3,163.0,149.4,136.9,134.0,129.7,129.2,125.4,124.5,115.1,107.6,58.0,55.7,41.3$,

21.2. HRMS (ESI-TOF) m/z: $[\mathrm{M}+\mathrm{H}]^{+}$Calcd for $\mathrm{C}_{17} \mathrm{H}_{18} \mathrm{NO}_{2}$ 268.1337; Found: 268.1344; $\left[\mathrm{M}+\mathrm{H}+\mathrm{CH}_{3} \mathrm{CN}\right]^{+}$Calcd for $\mathrm{C}_{19} \mathrm{H}_{21} \mathrm{~N}_{2} \mathrm{O}_{2}$ 309.1603; Found: 309.1610; $\left[\mathrm{M}+\mathrm{Na}+\mathrm{CH}_{3} \mathrm{CN}\right]^{+}$Calcd for $\mathrm{C}_{19} \mathrm{H}_{20} \mathrm{~N}_{2} \mathrm{O}_{2} \mathrm{Na} 331.1422$ Found: 331.1451.

3-(4-Trifluoromethyl-benzyl)-3-hydro-2,5-dimethoxy-isoindolin-1-one 4if. Colorless solid (45 mg, $0.128 \mathrm{mmol}, 43 \%) .{ }^{1} \mathrm{H}$ NMR $\left(\mathrm{CDCl}_{3}, 400 \mathrm{MHz}\right): \delta 7.62(\mathrm{~d}, 1 \mathrm{H}, J=8.4 \mathrm{~Hz}), 7.45$ (d, 2H, $\left.J=8.0 \mathrm{~Hz}\right)$, $7.21(\mathrm{~d}, 2 \mathrm{H}, J=8.0 \mathrm{~Hz}), 6.89(\mathrm{dd}, 1 \mathrm{H}, J=8.5,2.3 \mathrm{~Hz}), 6.51(\mathrm{~d}, 1 \mathrm{H}, J=2.3 \mathrm{~Hz}), 4.94(\mathrm{dd}, 1 \mathrm{H}, J=6.8,4.4$ $\mathrm{Hz}), 3.94(\mathrm{~s}, 3 \mathrm{H}), 3.75(\mathrm{~s}, 3 \mathrm{H}), 3.39$ (dd, $1 \mathrm{H}, J=14.0,4.4 \mathrm{~Hz}), 3.17$ (dd, $1 \mathrm{H}, J=14.0,6.8 \mathrm{~Hz}) .{ }^{13} \mathrm{C} \mathrm{NMR}$ $\left(\mathrm{CDCl}_{3}, 101 \mathrm{MHz}\right): \delta 165.1,163.0,143.3,139.5,130.2,125.6,125.3(\mathrm{q}, J=3.6 \mathrm{~Hz}), 125.0,122.2,115.0$, 108.2, 63.9, 59.8, 55.6, 37.5. HRMS (ESI-TOF) m/z: $[\mathrm{M}+\mathrm{H}]^{+}$Calcd for $\mathrm{C}_{18} \mathrm{H}_{17} \mathrm{NO}_{3} \mathrm{~F}_{3}$ 352.1160; Found: 352.1154; $\left[\mathrm{M}+\mathrm{H}+\mathrm{CH}_{3} \mathrm{CN}\right]^{+}$Calcd for $\mathrm{C}_{20} \mathrm{H}_{20} \mathrm{~N}_{2} \mathrm{O}_{3} \mathrm{~F}_{3}$ 393.1426; Found: 393.1397 .

3-(4-Trifluoromethyl-benzyl)-2,3-dihydro-5-methoxy-isoindolin-1-one, (4'if). Colorless solid (8 mg, $0.024 \mathrm{mmol}, 8 \%)$. mp $142-143{ }^{\circ} \mathrm{C} .{ }^{1} \mathrm{H}$ NMR $\left(\mathrm{CDCl}_{3}, 400 \mathrm{MHz}\right): \delta 7.75(\mathrm{~d}, 1 \mathrm{H}, J=8.4 \mathrm{~Hz}), 7.63-7.57$ (m, 2H), 7.38-7.33 (m, 2H), 7.00 (dd, 1H, $J=8.5,2.2 \mathrm{~Hz}), 6.80-6.75(\mathrm{~m}, 1 \mathrm{H}), 6.32$ (br s, 1H), 4.94 (dd, $1 \mathrm{H}, J=10.9,2.4 \mathrm{~Hz}), 3.85(\mathrm{~s}, 3 \mathrm{H}), 3.27(\mathrm{dd}, 1 \mathrm{H}, J=13.6,5.1 \mathrm{~Hz}), 2.89(\mathrm{dd}, 1 \mathrm{H}, J=13.6,8.8 \mathrm{~Hz}) .{ }^{13} \mathrm{C}$ $\operatorname{NMR}\left(\mathrm{CDCl}_{3}, 101 \mathrm{MHz}\right): \delta 170.3,163.2,148.8,141.1,129.8,125.9(\mathrm{q}, J=3.6 \mathrm{~Hz}), 125.6,124.4,115.2$, 107.7, 57.3, 55.8, 41.5. HRMS (ESI-TOF) m/z: $[\mathrm{M}+\mathrm{H}]^{+}$Calcd for $\mathrm{C}_{17} \mathrm{H}_{15} \mathrm{NO}_{2} \mathrm{~F}_{3}$ 322.1055; Found: 322.1062; $\left[\mathrm{M}+\mathrm{H}+\mathrm{CH}_{3} \mathrm{CN}\right]^{+}$Calcd for $\mathrm{C}_{19} \mathrm{H}_{18} \mathrm{~N}_{2} \mathrm{O}_{2} \mathrm{~F}_{3} 363.1320$; Found: 363.1333 .

\section{Acknowledgment}

The work was performed during CKX's sabbatical sojourn at Imperial College London as a Visiting Researcher. The authors are grateful to National Natural Science Foundation of China (No. 21171075) 
for financial support during this period. We also acknowledge Johnson Matthey PLC for supplying Pd salts.

Supporting information: Additional experimental results, copies of NMR spectra, ORTEP diagrams and X-ray crystallographic data (CIF).

\section{References}

1. International Patent, WO2013/120835A1, 2013.

2. International Patent PCT/CN2011/083229, WO2013/079452, 2013.

3. Valencia, E.; Freyer, A. J.; Shamma, M.; Fajardo, V. Tetrahedron Lett. 1984, 25, 599-602.

4. Desai, S. J.; Chaturvedi, R.; Mulchandani, N. B. J. Nat. Prod., 1990, 53, 496-497.

5. Choi, Y. L.; Kim, J. K.; Choi, S. U.; Min, Y. K.; Bae, M. A.; Kim, B. T.; Heo, J. N. Bioorg. Med. Chem. Lett. 2009, 19, 3036-3040.

6. (a) Rakshit, S.; Grohmann, C.; Besset, T.; Glorius, F. J. Am. Chem. Soc. 2011, 133, 2350-2353; b) Patureau, F. W.; Besset, T.; Glorius, F. Angew. Chem. Int. Ed. 2011, 50, 1064-1067; c) Fabry, D. C.; Zoller, J.; Raja, S.; Rueping, M. Angew. Chem. Int. Ed. 2014, 53, 10228-10231.

7. (a) Yang, F.; Ackermann, L. J. Org. Chem. 2014, 79, 12070-12082; b) Manoharan, R.; Jeganmohan, M. Chem. Commun. 2015, 51, 2929-2932.

8. Kim, J.; Park, S. W.; Baik, M. H.; Chang, S. J. Am. Chem. Soc. 2015, 137, 13448-13451.

9. Wrigglesworth, J. W.; Cox, B.; Lloyd-Jones, G. C.; Booker-Milburn, K. I. I. Org. Lett. 2011, 13, 53265329. 
10. Li, D. D.; Yuan, T. T.; Wang, G. W. Chem. Commun. 2011, 47, 12789-12791.

11. Das, A.; Chakrabarty, S.; Choudhury, D.; Chakrabarti, G. Chem. Res. Toxicol. 2010, 23, 1054-1066.

12. Fisher, L. E.; Caroon, J. M.; Jahangir, S.; Stabler, R.; Lundberg, S.; Muchowski, J. M. J. Org. Chem. 1993, $58,3643-3647$.

13. (a) Terao, Y.; Wakui, H.; Satoh, T.; Miura, M.; Nomura, M. J. Am. Chem. Soc. 2001, 123, 1040710408; (b) Bour, J. R.; Green, J. C.; Winton, V. J.; Johnson, J. B. J. Org. Chem. 2013, 78, 1665-1669.

14. Li, X. G.; Sun, M.; Liu, K.; Liu, P. N. Adv. Synth. Catal. 2015, 357, 395-399.

15. CrysAlis V1.171.34.44, Oxford Diffraction Ltd., Poland, 2010.

16. G. M. Sheldrick, SHELXL-97, Program for Crystal Structure Refinement; Göttingen University, Germany, 1997.

17. Fukui, Y.; Liu, P.; Liu, Q.; He, Z. T.; Wu, N. Y.; Tian, P.; Lin, G. Q. J. Am. Chem. Soc. 2014, 136, $15607-15614$. 